\title{
ASYMPTOTIC DISTRIBUTON OF EIGENVALUES AND EIGENFUNCTIONS FOR GENERAL LINEAR ELLIPTIC BOUNDARY VALUE PROBLEMS
}

\author{
BY \\ BUI AN TON
}

The asymptotic distribution of eigenvalues and eigenfunctions of elliptic operators has been studied extensively by Weyl, Courant, Carleman, Pleijel and others. During the last decade, Garding [9] and Browder solved the problem for an elliptic operator with infinitely differentiable coefficients and null Dirichlet boundary conditions. It is the purpose of this paper to consider the problem for a general class of elliptic boundary value problems investigated during the last few years by Agmon-Douglis-Nirenberg [2], Browder [3], [6] and Schechter [14].

Let $(A, \gamma)$ with $\gamma=\left\{B_{1}, \cdots, B_{m}\right\}$ be a uniformly regularly elliptic boundary value problem on $S$. It is assumed that $A$ is positively strongly elliptic and $(A, \gamma)$ is formally positive. If $A_{\gamma}$ is the realization of $A$ as an operator on $L^{2}(S)$ with null boundary conditions $\gamma$, then the following results are obtained:

(1) When $A_{\gamma}$ is self-adjoint:

$$
N(t)=\sum_{\lambda_{j} \leqq t} 1 \sim(2 \pi)^{-n} t^{n / 2 m} \int_{S} \int_{a(x, \xi)<1} d \xi d x \text { as } t \rightarrow+\infty .
$$

Let $e(x, y, t)$ be the spectral function of $A_{\gamma}$, then:

as $t \rightarrow+\infty ; x, y$ in $S$ and $x \neq y$.

$$
t^{-(n+|\alpha|+|\beta|) / 2 m} D_{x}^{\alpha} D_{y}^{\beta} e(x, y, t)=t^{-(n+|\alpha|+|\beta|) / 2 m} \sum_{\lambda_{j} \leqq t} D^{\alpha} \phi_{j}(x) D^{\beta} \phi_{j}(y) \rightarrow 0
$$

$$
\begin{array}{r}
D^{\alpha+\beta} e(x, x, t) \sim(2 \pi)^{-n} t^{(n+|\alpha|+|\beta|) / 2 m} \frac{\Gamma(2 p)}{\Gamma\left(1+\frac{n}{2 m}\right) \Gamma\left(2 p-\frac{n+|\alpha|+|\beta|}{2 m}\right)} \\
\cdot \int_{E^{n}} \xi^{\alpha+\beta}[a(x, \xi)+1]^{-2 p} d \xi
\end{array}
$$

for $x$ in $S$ as $t \rightarrow+\infty(4 m p>n+|\alpha|+|\beta|)$.

Received by the editors May 6, 1965.

(1) This is essentially a Doctoral Dissertation written under the guidance of Professor Felix Browder and submitted to the Department of Mathematics, Massachusetts Institute of Technology in June 1964. I would like to express my deep gratitude to Professor Browder for suggesting the problem, for his generous advices and encouragements. 
$\lambda_{j}, \phi_{j}$ are the eigenvalues and eigenfunctions of $A_{\gamma}$.

(2) When $A_{\gamma}$ is nonself-adjoint, then:

$$
N(t)=\sum_{\operatorname{Re} \lambda_{j} \leqq t} 1 \sim(2 \pi)^{-n} t^{n / 2 m} \int_{S} \int_{a(x, \xi)<1} d \xi d x \text { as } t \rightarrow+\infty .
$$

In $\S 1$, we give the notations and definitions; in $\S 2$, we state some known results; in $\$ 3$ the Green's function of $(A, \gamma)$ corresponding to the case of a half-space and constant coefficients is constructed. In $\$ 4$, using the parametrix method, we construct the Green's function associated with $(A, \gamma)$ when $A$ and $B_{j}$ are defined on $S, \Gamma$ and have infinitely differentiable coefficients. Results for the self-adjoint case are given in $\$ 5$ and for the nonself-adjoint case in $\$ 6$.

1. Notations and definitions. Let $E^{n}$ be the $n$-dimensional Euclidean space, $S$ an open subset of $E^{n}$. The points of $E^{n}$ will be denoted by $x=\left(x_{1}, \cdots, x_{n}\right)$ and integration with respect to $d x$ over a subset of $E^{n}$ denotes integration with respect to Lebesgue $n$-measure.

For $1 \leqq j \leqq n, D_{j}=i^{-1} \partial / \partial x_{j}$. If $\alpha=\left(\alpha_{1}, \cdots, \alpha_{n}\right)$ is any $n$-tuple of nonnegative integers, we set:

$$
|\alpha|=\sum_{j=1}^{n} \alpha_{j}, \quad D^{\alpha}=\prod_{j=1}^{n} D_{j}^{\alpha} .
$$

The linear partial differential operator $A=\Sigma_{|\alpha| \leqq 2 m} a_{\alpha}(x) D^{\alpha}$ with coefficients defined on $S$ is said to be uniformly elliptic on $S$ if there exists a constant $c>0$ such that:

$$
|a(x, \xi)| \geqq c|\xi|^{2 m} \text { for every point } \xi \in R^{n} \text {. }
$$

$a(x, \xi)$ is the characteristic form of $A$ and is given by:

$$
a(x, \xi)=\sum_{|\alpha|=2 m} a_{\alpha}(x) \xi^{\alpha}, \quad \xi \text { in } R^{n} .
$$

We shall assume throughout for the sake of simplicity that $S$ is bounded and that its boundary is locally a $C^{\infty},(n-1)$-dimensional manifold with $C^{\infty}$ imbedding in $E^{n}$. In particular, $S$ will satisfy the uniform regularity conditions (Browder [5]).

DeFINITION 1.1. Let $j$ be a nonnegative integer:

$$
W^{j, 2}(S)=\left\{u: u \in L^{2}(S) ; D^{\alpha} u \in L^{2}(S),|\alpha| \leqq j\right\},
$$

where $D^{\alpha} u$ denotes the derivative in the distribution sense.

$W^{j, 2}(S)$ is a Hilbert space with respect to the inner product:

$$
\begin{aligned}
& (u, v)_{j}=\sum_{|\alpha| \leqq j}\left(D^{\alpha} u, D^{\alpha} v\right), \quad u, v \text { in } W^{j, 2}(S), \\
& (u, v)=\int_{S} u(x) \overline{v(x)} d x
\end{aligned}
$$

is the usual inner product in $L^{2}(S)$. 
The norm in $W^{j, 2}(S)$ is given by:

$$
\|u\|_{j, 2}=\left(\sum_{|\alpha| \leqq j}\left\|D^{\alpha} u\right\|_{L^{2}(S)}^{2}\right)^{1 / 2} .
$$

Let $\gamma=\left(B_{1}, \cdots, B_{m}\right)$ be a family of $m$ differential operators with coefficients defined on $S$. We assume that the order $r_{j}$ of each $B_{j}$ is less than $2 m$ and we write:

$$
B_{j}=\sum_{|\beta| \leqq r_{j}} b_{j \beta}(x) D^{\beta} .
$$

We also assume that the coefficients $b_{j \beta}$ lie in $C^{\infty}(\bar{S})$. For each $j$, the characteristic form is defined for $\zeta \in C^{n}$ by

$$
b_{j}(x, \zeta)=\sum_{|\beta|=r_{j}} b_{j \beta}(x) \zeta^{\beta} .
$$

DEFINITION 1.2. $A$ is said to be regularly elliptic on $S$ if it is uniformly elliptic on $S$ and if for each $x$ of $\Gamma$, the polynomial $a\left(x, \lambda N_{x}+T\right)$ in $\lambda\left(\lambda \in C ; N_{x}\right.$ is the unit exterior normal vector to $\Gamma$ at $x, T$ any unit tangent vector to $\Gamma$ at $x$ ) has exactly $m$ roots (counting multiplicities) in the $\lambda$ upper half plane.

DeFINITION 1.3. The boundary value problem $(A, \gamma)$ is said to be uniformly regular if:

(1) $A$ is uniformly regularly elliptic.

(2) For each unit tangent vector $T$ to $\Gamma$ at $x$, let $C_{T}$ be a closed, Jordan rectifiable curve in the $\lambda$ half plane which contains in its interior all the zeros of $a\left(x, \lambda N_{x}+T\right)$ with positive imaginary parts.

For $1 \leqq j, k \leqq m ;$ set :

$$
c_{j k}(x, T)=\int_{C_{T}} \lambda^{j-1} b_{k}\left(x, \lambda N_{x}+T\right)\left[a\left(x, \lambda N_{x}+T\right)\right]^{-1} d \lambda .
$$

Then there exists $c>0$ such that:

$$
\left|\operatorname{Det}\left(c_{j k}(x, T)\right)\right| \geqq c>0
$$

for all $x \in \Gamma$ and all unit tangent vectors to $\Gamma$ at $x$.

2. Definition 2.1. Let $x_{0}$ be a point of $\Gamma ; A_{0}$ the homogeneous differential operator of order $2 m$ with constant coefficients

$$
A_{0}=\sum_{|\alpha|=2 m} a_{\alpha}\left(x_{0}\right) D^{\alpha} .
$$

Let $\gamma_{0}=\left(B_{10}, \cdots, B_{m 0}\right)$ where:

$$
B_{j 0}=\sum_{|\beta|=r_{j}} b_{j \beta}\left(x_{0}\right) D^{\beta} .
$$

Then $(A, \gamma)$ is said to be formally positive if the boundary value problem: 
1966] ASYMPTOTIC DISTRIBUTION OF EIGENVALUES AND EIGENFUNCTIONS 519

$$
\begin{aligned}
\left(A_{0}+t I\right) u & =f \text { on } S, & & t>0, \\
B_{j} u & =0 \text { on } \Gamma, & & j=1, \cdots, m,
\end{aligned}
$$

has a unique solution $u$ in $C^{\infty}(S) \cap L^{2}(S)$ for every $f$ in $\left.C_{c}^{\infty}\left(E^{n}\right)\right|_{s}$ and $t>0$, such that:

$$
\|u\|_{L^{2}(S)} \leqq c\|f\|_{L^{2}(S)}
$$

with the constant $c$ independent of $u, f$.

We shall take the above definition as our basic assumption on $(A, \gamma)$. Alternative assumptions which are equivalent to Definition 2.1 may be given in a more computational form; e.g. as given by Agmon [1]: the polynomial in the complex variable $\lambda, a\left(x_{0}, \lambda N_{x_{0}}+T\right)+t$ has no real roots; it has $m$ roots with positive imaginary parts and:

$$
\begin{aligned}
&\left|\operatorname{Det}\left(\int_{C_{t}, T} \lambda^{j-1} b_{r}\left(x_{0}, \lambda N_{x_{0}}+T\right)\left[a\left(x_{0}, \lambda N_{x_{0}}+T\right)+t\right]^{-1} d \lambda\right)\right|>0, \\
& r, j=1, \cdots, m .
\end{aligned}
$$

$C_{t, T}$ is a closed Jordan rectifiable curve surrounding all the roots with positive imaginary parts of $a\left(x_{0}, \lambda N_{x_{0}}+T\right)+t$ considered as a polynomial in $\lambda$.

DEFINITION 2.2. (1) Let $A_{\gamma}$ be the operator on $L^{2}(S)$ defined as follows:

$$
\begin{aligned}
D\left(A_{\gamma}\right) & =\left\{u: u \text { in } W^{2 m, 2}(S) ; B_{j} u=0 \text { on } \Gamma ; j=1, \cdots, m\right\}, \\
A_{\gamma} u & =A u \text { if } u \in D\left(A_{\gamma}\right) .
\end{aligned}
$$

(2) $(A, \gamma)$ is said to be formally self-adjoint if $A_{\gamma}$ is a symmetric operator in $L^{2}(S)$; i.e.

$$
\left(A_{\gamma} u, v\right)=\left(u, A_{\gamma} v\right)
$$

for all $u, v$ in $D\left(A_{\gamma}\right)$.

THEOREM 2.1. Let $(A, \gamma)$ be a uniformly regularly elliptic boundary value problem as above, such that $A$ is uniformly strongly elliptic and $(A, \gamma)$ is formally positive.

Then:

(1) If $(A, \gamma)$ is formally self-adjoint; $A_{\gamma}$ is then a self-adjoint operator on $L^{2}(S)$.

(2) If $t \geqq t_{0}>0 ;\left(A_{\gamma}+t I\right)^{-1}$ exists on all of $L^{2}(S)$ and:

$$
\left\|\left(A_{\gamma}+t I\right)^{-1}\right\| \leqq c t^{-1} .
$$

(3) If $p$ is a positive integer such that $2 m p>n ;\left(A_{\gamma}+t I\right)^{-p}$ is an operator of Hilbert-Schmidt type, 


$$
\left(A_{\gamma}+t I\right)^{-p} f(x)=\int_{S} \mathscr{G}_{(p)}(x, y, t) f(y) d y \quad f \text { in } L^{2}(S)
$$

and $\mathscr{G}_{(p)}(x, y, t) \in L^{2}(S) \times L^{2}(S)$.

Proof. It has been proved by Agmon [1] that when $(A, \gamma)$ is formally positive then $\left\|\left(A_{\gamma}+t I\right)^{-1}\right\| \leqq c t^{-1}$ and moreover if $A_{\gamma}$ is formally self-adjoint, then $A_{\gamma}$ is self-adjoint.

Since $(A, \gamma)$ is a regular elliptic boundaryvalue problem; we have the a priori estimate:

$$
\begin{aligned}
&\|u\|_{W^{2 m p, 2}} \leqq C\left\{\|u\|_{L^{2}}+\left\|\left(A_{\gamma}+t I\right) u\right\|^{W^{2 m(p-1), 2}}\right\}, \\
&\|u\|_{W^{2 m p, 2}} \leqq C\left\|\left(A_{\gamma}+t I\right)^{p} u\right\|_{L^{2}} .
\end{aligned}
$$

Therefore $\left(A_{\gamma}+t I\right)^{-p}$ is a continuous mapping of $L^{2}(S)$ into $W^{2 m p, 2}(S)$. From the Sobolev imbedding theorem, the injection mapping:

$$
W^{2 m p, 2}(S) \rightarrow L^{\infty}(S)
$$

is continuous when $2 m p>n$. Hence $\left(A_{\gamma}+t I\right)^{-p}$ considered as a mapping of $L^{2}(S)$ to $L^{\infty}(S)$ is continuous. By the Dunford-Pettis theorem, it follows that $\left(A_{\gamma}+t I\right)^{-p}$ is of Hilbert-Schmidt type:

$$
\left(A_{\gamma}+t I\right)^{-p} f(x)=\int_{S} \mathscr{G}_{(p)}(x, y, t) f(y) d y, f \text { in } L^{2}(S) .
$$

3. Let $A$ be a homogeneous linear elliptic differential operator on $E^{n}$ with constant coefficients, of order $2 m$.

Let $\gamma=\left\{B_{j}, j=1, \cdots, m\right\}$ be a family of homogeneous linear differential operators defined on $E^{n}$ with constant coefficients and of order $r_{j}<2 m-1$. Let $t$ be a positive parameter such that $(A, \gamma)$ is formally positive in the sense of Definition 2.1. In this section, we construct: (1) the Green's function $G(x, y, t)$ associated with $(A+t I, \gamma)$; (2) the iterates of $G$. Finally we study the asymptotic behavior of $G_{(p)}$ as $t \rightarrow+\infty$.

LEMMA 3.1. Let $A$ be a homogeneous, linear elliptic operator of order $2 m$, on $E^{n}$ with constant coefficients. Let $t$ be a positive parameter such that $A+t I$ is positively strongly elliptic. Then $A+t I$ has a fundamental solution $E(x, y, t)$ given by:

$$
E(x, y, t)=(2 \pi)^{-n} \int_{E^{n}} \exp (i\langle x-y, \xi\rangle)[a(\xi)+t]^{-1} d \xi
$$

where the integral is taken as the Fourier transform of a tempered distribution if $2 m<n$.

$E(x, y, t)$ is infinitely differentiable for $x \neq y$ and if $t=\tau^{2 m}$ then: 
1966] ASYMPTOTIC DISTRIBUTION OF EIGENVALUES AND EIGENFUNCTIONS 521

$$
D^{\alpha} E(x, y, t)=O(1) \tau^{-8}|x-y|^{-n+2 m-|\alpha|-\varepsilon}\left(1+|\tau(x-y)|^{N}\right)^{-1}
$$

if $-n+2 m-|\alpha| \leqq 0$ and

$$
D^{\alpha} E(x, y, t)=O(1) \tau^{-\varepsilon}\left(1+|\tau(x-y)|^{N}\right)^{-1}
$$

if $-n+2 m-|\alpha|>0$, where $0<\varepsilon<1 ; N$ is an arbitrary positive number (Gårding [9]).

THEOREM 3.1. Let $v$ be the solution of the boundary value problem:

$$
\begin{aligned}
(A+t I) v & =0 \text { on } E_{+}^{n}, \\
B_{j} v & =B_{j} E \text { on } E^{n-1}, \quad j=1, \cdots, m,
\end{aligned}
$$

where $(A, \gamma)$ is a regular elliptic boundary value problem; $A$ and $B_{j}$ are homogeneous and have constant coefficients. Then:

(1) $v(x, y, t)$ is infinitely differentiable for $x \neq y$ and is given by:

with:

$$
v(x, y, t)=\sum_{r, j=1}^{m}(2 \pi)^{-n} \int_{E^{n-1}} \exp (i\langle\hat{x}-\hat{y}, \hat{\xi}\rangle) V_{r j}\left(\hat{\xi}, x_{1}, y_{1}, t\right) d \hat{\xi}
$$

$$
V_{r j}\left(\hat{\xi}, x_{1}, y_{1}, t\right)=Q_{r j}(\hat{\xi}, t) H_{j}\left(\hat{\xi}, y_{1}, t\right) \int_{C_{\xi, t}} \zeta_{1}^{r-1} \exp \left(i \zeta_{1} x_{1}\right)\left[a\left(\zeta_{1}, \hat{\xi}\right)+t\right]^{-1} d \zeta_{1}
$$

where $C_{\xi, t}$ is a closed, Jordan rectifiable curve in the $\zeta_{1}$ upper half plane and surrounding all the $m$ roots (counting multiplicities) of $a\left(\zeta_{1}, \hat{\xi}\right)+t=0$ for fixed $\hat{\xi}, t$.

$$
H_{j}\left(\hat{\xi}, y_{1}, t\right)=\int_{E^{1}} \exp \left(-i y_{1} \xi_{1}\right) b_{j}\left(\xi_{1}, \hat{\xi}\right)\left[a\left(\xi_{1}, \hat{\xi}\right)+t\right]^{-1} d \xi_{1}
$$

Finally $Q_{r j}$ are the elements of the transpose of the inverse of the matrix $\left(c_{r j}(\hat{\xi}, t)\right)$ with:

$$
c_{r j}(\xi, t)=\int_{C \xi_{t},} \zeta_{1}^{r-1} b_{j}\left(\zeta_{1}, \hat{\xi}\right)\left[a\left(\zeta_{1}, \hat{\xi}\right)+t\right]^{-1} d \zeta_{1}, \quad r, j=1, \cdots, m .
$$

(2) Let $t=\tau^{2 m}$ then $D^{\alpha} v(x, y, t)=O(1) \tau^{-\varepsilon}|\hat{x}-\hat{y}|^{-n+2 m-|\alpha|-\varepsilon}\left(1+|\hat{x}-\hat{y}|^{N}\right)^{-1}$ if $-n+2 m-|\alpha| \leqq 0$ and $D^{\alpha} v=O(1) \tau^{-\varepsilon}\left(1+|\hat{x}-\hat{y}|^{N}\right)^{-1}$ if $n+2 m-|\alpha|>0$; $0<\varepsilon<1 ; N$ is an arbitrary positive number.

Proof. We write:

$$
A=\sum_{q=0}^{2 m} A_{q}(\hat{D}) D_{1}^{q}, \quad B_{j}=\sum_{q=0}^{r j} B_{j q}(\hat{D}) D_{1}^{q},
$$

where $\hat{D}$ denotes differential operators involving only $D_{2}, \cdots, D_{n}$. Taking the 
Fourier transform with respect to the tangential variables $\hat{x}=\left(x_{2}, \cdots, x_{n}\right)$ we are reduced to the initial value problem:

$$
\begin{aligned}
\left(\sum_{q=0}^{2 m} a_{q}(\hat{\xi}) D_{1}^{q}+t I\right) V\left(x_{1}, \hat{\xi}\right) & =0, \quad x_{1}>0, \\
& \sum_{q=0}^{r j} b_{j q}(\hat{\xi}) D_{1}^{q} V\left(x_{1}, \hat{\xi}\right)=H_{j}(\hat{\xi}, t) ; \quad x_{1}=0 ; j=1, \cdots, m,
\end{aligned}
$$

where:

$$
V\left(x_{1}, \hat{\xi}\right)=(2 \pi)^{-(n-1) / 2} \int_{E^{n-1}} \exp (-i\langle\hat{x}, \hat{\xi}\rangle) v\left(\hat{x}_{1}, \hat{x}\right) d \hat{x}
$$

$H_{j}(\hat{\xi}, t)$ is similarly defined $\left(r_{j}<2 m-1\right)$.

We consider a solution of the form

$$
V\left(x_{1}, \hat{\xi}\right)=(2 \pi)^{-1} \int_{c_{\xi, t}} \exp \left(i x_{1} \zeta_{1}\right)\left[a\left(\zeta_{1}, \hat{\xi}\right)+t\right]^{-1} p\left(\zeta_{1}\right) d \zeta_{1},
$$

where $p\left(\zeta_{1}\right)$ is a polynomial of degree less than or equal to $2 m$ and $C_{\xi, t}$ is a closed, Jordan rectifiable curve in the upper half plane surrounding all the $m$ roots (counting multiplicities) of $a\left(\zeta_{1}, \hat{\xi}\right)+t=0$ for fixed $\hat{\xi}, t$.

By Cauchy's theorem, we may assume that $p\left(\zeta_{1}\right)$ is a polynomial of degree less than $m$ :

$$
p\left(\zeta_{1}\right)=\sum_{r=1}^{m} p_{r} \zeta_{1}^{r-1}
$$

We obtain a system of $m$ equations with $m$ unknowns.

$$
(2 \pi)^{-1} \sum_{r=1}^{m} p_{r}(\hat{\xi}) \int_{c_{\xi, t}} \zeta_{1}^{r-1} b_{j}\left(\zeta_{1}, \hat{\xi}\right)\left[a\left(\zeta_{1}, \hat{\xi}\right)+t\right]^{-1} d \zeta_{1}=H_{j}(\hat{\xi}, t) ; \quad j=1, \cdots, m .
$$

We may solve it in a unique fashion. Indeed, we have:

$$
\begin{aligned}
& \operatorname{Det}\left(\int_{\xi, t} \zeta_{1}^{r-1} b_{j}\left(\zeta_{1}, \hat{\xi}\right)\left[a\left(\zeta_{1}, \hat{\xi}^{\prime}\right)+t\right]^{-1} d \zeta_{1}\right) \\
& \quad=|\hat{\zeta}|^{\Sigma_{j^{\prime}}+\left(m-m^{2}\right) / 2} \operatorname{Det}\left(\int_{C_{\xi, t,\left|\xi^{\prime}\right|-m 2}} \zeta_{1}^{r-1} b_{j}\left(\zeta_{1}, \hat{\xi}^{\prime}\right)\left[a\left(\zeta_{1}, \hat{\xi}^{\prime}\right)+t\left|\hat{\xi}^{\prime}\right|^{-2 m}\right]^{-1} d \zeta_{1}\right)
\end{aligned}
$$

and the last expression is nonnull by hypothesis for $|\hat{\xi}| \neq 0$. We get:

$$
\begin{aligned}
p_{r}(\hat{\xi}) & =\sum_{j=1}^{m} Q_{r j}(\hat{\xi}, t) H_{j}(\hat{\xi}, t), \\
V\left(x_{1}, \hat{\xi}, t\right) & =\sum_{j=1}^{m} Q_{r j}(\hat{\xi}, t) H_{j}(\hat{\xi}, t) \int_{c_{\xi, t}} \zeta_{1}^{r-1} \exp \left(i \zeta_{1} x_{1}\right)\left[a\left(\zeta_{1}, \hat{\xi}\right)+t\right]^{-1} d \zeta_{1} .
\end{aligned}
$$


We want to take the inverse Fourier transform of $V$. To prove the summability of $V\left(x_{1}, \hat{\xi}, t\right)$ with respect to $\hat{\xi}$ we establish the following lemmas.

LEMMA 3.2. The following estimates hold uniformly for $t \geqq t_{0}>0$ :

$$
\begin{aligned}
f\left(x_{1}, \hat{\xi}, t\right) & =\int_{C_{\xi, t}} \zeta_{1}^{r-1} \exp \left(i \zeta_{1} x_{1}\right)\left[a\left(\zeta_{1}, \hat{\xi}\right)+t\right]^{-1} d \zeta_{1} \\
& =O\left(\left(1+|\hat{\xi}|^{2 m-r}\right)^{-1}\right) \exp \left(-d|\hat{\xi}| x_{1}\right)
\end{aligned}
$$

where $x_{1}>0, d>0$ independent of $t$ and $C_{\xi, t}$ is a closed, Jordan rectifiable curve in thc $\zeta_{1}$ upper half plane surrounding all the $m$ roots of $a\left(\zeta_{1}, \hat{\xi}\right)+t$ for fixed $\hat{\xi}, t . a\left(\zeta_{1}, \hat{\xi}\right)$ is a homogeneous polynomial of degree $2 \mathrm{~m}$.

Proof. Making the change of variables $\hat{\xi}=t^{1 / 2 m} \hat{\xi}^{\prime}, \zeta_{1}=t^{1 / 2 m \zeta_{1}^{\prime}}$, we get:

We consider:

$$
\begin{aligned}
f\left(x_{1}, \hat{\xi}, t\right) & =t^{r / 2 m-1} \int_{C \xi^{\prime}, 1} \zeta_{1}^{r-1} \exp \left(i \zeta_{1} x_{1} t^{1 / 2 m}\right)\left[a\left(\zeta_{1}, \hat{\xi}\right)+1\right]^{-1} d \zeta_{1} \\
& =t^{r / 2 m-1} f\left(x_{1} t^{1 / 2 m}, \hat{\xi}^{-1 / 2 m}, 1\right) .
\end{aligned}
$$

$$
f\left(x_{1}, \hat{\xi}, 1\right)=\int_{c_{\xi}} \zeta_{1}^{r-1} \exp \left(i \zeta_{1} x_{1}\right)\left(a\left(\zeta_{1}, \hat{\xi}\right)+1\right]^{-1} d \zeta_{1} .
$$

(1) the equation $a\left(\zeta_{1}, \hat{\xi}\right)+1=0$ may be written as $c \zeta_{1}^{2 m}+P\left(\zeta_{1}, \hat{\xi}\right)=0$ where $P\left(\zeta_{1}, \hat{\xi}\right)$ is a polynomial of degree less than or equal to $2 m-1$ in the $\zeta_{1}$ variable and such that $P\left(\zeta_{1}, 0\right)=1 ; c$ is a constant. The roots of $a\left(\zeta_{1}, \hat{\xi}\right)+1=0$ depend continuously on the parameter $\hat{\xi}$ and as $\hat{\xi}$ goes to zero they go to the roots of $c \zeta_{1}^{2 m}+1=0$. Therefore, there is a closed curve $C$ containing all the roots of $a\left(\zeta_{1}, \hat{\xi}\right)+1=0$ for fixed and small $\hat{\xi}$.

For $|\hat{\xi}|<1$, we have:

$$
f\left(x_{1}, \hat{\xi}, 1\right)=\int_{C} \zeta_{1}^{r-1} \exp \left(i x_{1} \zeta_{1}\right)\left[a\left(\zeta_{1}, \hat{\xi}\right)+1\right]^{-1} d \zeta_{1}
$$

so:

$$
\begin{aligned}
& \left|f\left(x_{1}, \hat{\xi}, 1\right)\right| \leqq M, \\
& \left|f\left(x_{1}, \hat{\xi}, t\right)\right| \leqq M t_{0}^{r / 2 m-1} .
\end{aligned}
$$

(2) To study the behavior of $f\left(x_{1}, \hat{\xi}, 1\right)$ as $|\hat{\xi}|$ goes to infinity, we make the change of variables $\hat{\xi}=\tau \hat{\xi}^{\prime} ; \zeta_{1}=\tau \zeta_{1}$ with $\tau=|\hat{\xi}|$. Then

$$
f\left(x_{1}, \hat{\xi}, 1\right)=|\hat{\xi}|^{r-2 m} \int_{C_{\xi, t-2 m}} \exp \left(i \zeta_{1} \tau x_{1}\right)\left[a\left(\zeta_{1}, \hat{\xi}^{\prime}\right)+\tau^{-2 m}\right]^{-1} d \zeta_{1} .
$$

Consider the equation $a\left(\zeta_{1}, \hat{\xi}^{\prime}\right)+\tau^{-2 m}=0$ for large $\tau$. The roots of the equation considered as a polynomial in $\zeta_{1}$ depend continuously on $\tau^{-1}$ and so as $\tau$ goes to infinity they go to the roots of $a\left(\zeta_{1}, \hat{\xi}^{\prime}\right)=0 ;\left|\hat{\xi}^{\prime}\right|=1$. Therefore there exists 
a closed curve $C_{\xi^{\prime}}$ in the upper half plane surrounding all the roots of $a\left(\zeta_{1}, \hat{\xi}^{\prime}\right)+\tau^{-2 m}=0$ for large $\tau$

$$
f\left(x_{1}, \xi, 1\right)=\tau^{r-2 m} \int_{C_{\xi^{\prime}}} \zeta_{1}^{r-1} \exp \left(i \zeta_{1} \tau x_{1}\right)\left[a\left(\zeta_{1}, \hat{\xi}^{\prime}\right)+\tau^{-2 m}\right]^{-1} d \zeta_{1} .
$$

On the curve $C_{\xi^{\prime}}$, we have, $\left|a\left(\zeta_{1}, \hat{\xi}\right)\right| \geqq c>0$. On the other hand $\left|a\left(\zeta_{1}, \hat{\xi}^{\prime}\right)+\tau^{-2 m}\right| \geqq\left|a\left(\zeta_{1}, \hat{\xi}^{\prime}\right)\right|-\tau^{-2 m}$. For sufficiently large $\tau,\left|a\left(\zeta_{1}, \hat{\xi}^{\prime}\right)+\tau^{-2 m}\right|$ $\geqq c_{1}>0$. Therefore for large $\hat{\xi}$

$$
\left|f\left(x_{1}, \hat{\xi}, 1\right)\right| \leqq M \exp \left(-d|\hat{\xi}| x_{1}\right)|\hat{\xi}|^{r-2 m}
$$

where $d=\operatorname{Inf}_{\zeta_{1} \in C \xi^{\prime}}\left(\operatorname{Im} \zeta_{1}\right)>0, M$ is a constant and

$$
\left|f\left(x_{1}, \hat{\xi}, t\right)\right| \leqq M \exp \left(-d|\hat{\xi}| x_{1}\right)|\hat{\xi}|^{r-2 m}, \quad|\hat{\xi}|>1 \text {. }
$$

$M$ is independent of $x_{1}, t$.

Combining (1), (2) we get the lemma.

LEMMA 3.3. 1. The following estimates hold uniformly for $t \geqq t_{0}>0$

$c_{r j}(\hat{\xi}, t)=\int_{C \xi, t} \zeta_{1}^{r-1} b_{j}\left(\zeta_{1}, \hat{\xi}\right)\left[a\left(\zeta_{1}, \hat{\xi}\right)+t\right]^{-1} d \zeta_{1}=O\left(\left(1+|\hat{\xi}|^{r+r_{j}}\right)\left(1+|\hat{\xi}|^{2 m}\right)^{-1}\right)$

where $r=1, \cdots, m ; r_{j}<2 m$ and $C_{\xi, t}$ is a closed Jordan rectifiable curve in the $\zeta_{1}$ upper half plane surrounding all the $m$ roots of $a\left(\zeta_{1}, \hat{\xi}\right)+t=0$ for fixed $\hat{\xi}, t . b_{j}\left(\zeta_{1}, \hat{\xi}\right) ; a\left(\zeta_{1}, \hat{\xi}\right)$ are homogeneous polynomials of degree $r_{j}, 2 m$ respectively.

2. Let $Q_{r j}(\xi, t)$ be the elements of the transpose of the inverse of the matrix $\left(c_{r j}(\hat{\xi}, t)\right)$ then:

$$
Q_{r j}(\xi, t)=O\left(\left(1+|\hat{\xi}|^{2 m}\right)\left(1+|\hat{\xi}|^{r+r_{J}}\right)^{-1}\right) .
$$

Proof. The proof is similar to that of Lemma 3.2. We will not repeat it.

We return to the proof of Theorem 3.1. From Lemmas 3.2, 3.3 and noting that $\left.H_{j}(\hat{\xi}, t)=O\left(1+|\hat{\xi}|^{r_{j}}\right)\left(1+|\hat{\xi}|^{2 m}\right)^{-1}\right)$ we obtain:

$$
V\left(x_{1}, \hat{\xi}, t\right)=O\left(\left(1+|\hat{\xi}|^{2 m}\right)^{-1}\right) \exp \left(-d|\hat{\xi}| x_{1}\right)
$$

where $d>0 ; x_{1}>0 ; t \geqq t_{0}>0$.

For $x_{1}>0, V\left(x_{1}, \hat{\xi}, t\right)$ is integrable with respect to $\xi$ we have:

$(2 \pi)^{n} v(x, t)=$

$\sum_{r, j=1}^{m} \int_{E^{n-1}} \exp (i\langle\hat{x}, \hat{\xi}\rangle) Q_{r j}(\hat{\xi}, t) H_{j}(\hat{\xi}, t) \int_{C_{\xi, t}} \zeta_{1}^{r-1} \exp \left(i \zeta_{1} x_{1}\right)\left[a\left(\zeta_{1}, \hat{\xi}\right)+t\right]^{-1} d \zeta_{1} d \hat{\xi}$

We study the regularity of $v(x, t)$. The results are stated in the following lemma:

LEMma 3.4. Let $v(x, t)$ be given by the expression: 
1966] ASYMPTOTIC DISTRIBUTION OF EIGENVALUES AND EIGENFUNCTIONS 525

$v(x, t)=$

$\sum_{r, j=1}^{m}(2 \pi)^{-} \int_{E^{n-1}} \exp (i\langle\hat{x}, \hat{\xi}\rangle) Q_{r j}(\hat{\xi}, t) H_{j}(\hat{\xi}, t) \int_{C \xi, t} \xi_{1}^{r-1} \exp \left(i \zeta_{1} x_{1}\right)\left[a\left(\zeta_{1}, \hat{\xi}\right)+t\right]^{-1} d \zeta_{1} d \hat{\xi}$

where $Q_{r j} ; H_{j}$ are defined in Theorem 3.1, then:

(1) $v(x, t)$ is infinitely differentiable for $\hat{x} \neq 0$.

(2) $D^{\alpha} v(0, \hat{x}, t)=O(1) t^{-\varepsilon / 2 m}|\hat{x}|^{-n-|\alpha|+2 m-\varepsilon}\left(1+|\hat{x}|^{N}\right)^{-1}$ if $-n-|\alpha|+2 m \leqq 0$ and $D^{\alpha} v(0, \hat{x}, t)=O(1) t^{-\varepsilon / 2 m}\left(1+|\hat{x}|^{N}\right)^{-1}$ otherwise. $0<\varepsilon<1$ and $N$ is an arbitrary positive integer.

Proof. (1) First we consider the case when $x_{1}>0$. We may take the differentiation under the integral sign. Indeed:

$K_{r j}\left(\hat{\xi}, x_{1}, t, \alpha+\beta\right)=\hat{\xi}^{\alpha} Q_{r j}(\hat{\xi}, t) H_{j}(\hat{\xi}, t) \int_{C_{\xi, t}} \zeta_{1}^{r-1+\beta} \exp \left(i \zeta_{1} x_{1}\right)\left[a\left(\zeta_{1}, \hat{\xi}\right)+t\right]^{-1} d \zeta_{1}$,

$K_{r j}\left(\hat{\xi}, x_{1}, t, \alpha+\beta\right)=O\left(|\hat{\xi}|^{\alpha+\beta}\left(1+|\hat{\xi}|^{2 m-1}\right)^{-1} \exp \left(-d|\hat{\xi}| x_{1}\right)\right)$,

hence for $x_{1}>0 ; v(x, t)$ is infinitely differentiable.

(2) We now study the regularity of $v(x, t)$ as $x_{1}$ goes to zero.

For $x_{1}>0$, we have:

$$
\hat{x}^{p} D^{\alpha+\beta} v\left(x_{1}, \hat{x}, t\right)=(2 \pi)^{-n} \sum_{r, j=1}^{m} \int_{E^{n-1}} \exp (i\langle\hat{x}, \hat{\xi}\rangle) D_{\xi}^{p} K_{r j}\left(\hat{\xi}, x_{1}, \alpha+\beta, t\right) d \hat{\xi} .
$$

Making the change of variables $\hat{\xi}=t^{1 / 2 m} \hat{\xi}^{\prime}, \zeta_{1}=t^{1 / 2 m} \zeta_{1}^{\prime}$, we get:

$(2 \pi)^{n} \hat{x}^{p} D^{\alpha+\beta} v\left(x_{1}, \hat{x}, t\right)$

$$
=\sum_{r, j=1}^{m} t^{(\alpha+\beta+n-p) / 2 m-1} \int_{E^{n-1}} \exp \left(i\left\langle\hat{x} t^{1 / 2 m}, \hat{\xi}\right\rangle\right) D_{\xi}^{p} K_{r j}\left(\hat{\xi}, x_{1} t^{1 / 2 m}, \alpha+\beta\right) d \hat{\xi} .
$$

Consider the expression $D_{\xi}^{p} K_{r j}\left(\hat{\xi}, x_{1} t^{1 / 2 m}, \alpha+\beta\right)$. We have:

$$
\left|D_{\xi}^{p} K_{r j}\left(\hat{\xi}, x_{1} t^{1 / 2 m}, \alpha+\beta\right)\right| \leqq M \text { for }|\hat{\xi}| \leqq 1 \text {. }
$$

$M$ is a constant independent of $x_{1}, t$.

Making the change of variables $\hat{\xi}=\tau \hat{\xi}^{\prime} ; \zeta_{1}=\tau \zeta_{1}^{\prime}$ with $\tau=|\hat{\xi}|$; we get:

$$
D_{\xi}^{p} K_{r j}\left(\hat{\xi}, x_{1} t^{1 / 2 m}, \alpha+\beta\right)=\tau^{\alpha+\beta+p-2 m+1} D_{\xi}^{p}, K_{r j}\left(\hat{\xi}^{\prime}, x_{1} t^{1 / 2 m} \tau, \alpha+\beta\right)
$$

so:

$$
\left|D_{\xi}^{p} K_{r j}\left(\hat{\xi}, x_{1} t^{1 / 2 m}, \alpha+\beta\right)\right| \leqq M|\hat{\xi}|^{|\alpha|+|\beta|-2 m+1-p} \text { for }|\hat{\xi}| \geqq 1 .
$$

If $|\alpha|+|\beta|+n+1-2 m \geqq 0$, we take $p=n+1+|\alpha|+|\beta|-2 m$ and:

$$
\left|D_{\xi}^{p} K_{r j}\left(\hat{\xi}, x_{1} t^{1 / 2 m}, \alpha+\beta\right)\right| \leqq M\left(1+|\hat{\xi}|^{n}\right)^{-1} \text { for all } \hat{\xi} \text {. }
$$

Since $\hat{x}^{p} D^{\alpha+\beta} v\left(x_{1}, \hat{x}, t\right)=0$ for $\hat{x}=0$ and $p=n+|\alpha|+|\beta|+1-2 m \geqq 0$, we obtain: 


$$
D^{\alpha+\beta} v\left(x_{1}, \hat{x}, t\right)=O(1) t^{-\varepsilon / 2 m}|\hat{x}|^{-n-|\alpha|-|\beta|+2 m-\varepsilon}\left(1+|\hat{x}|^{N}\right)^{-1} ; \quad x_{1} \geqq 0 .
$$

If $|\alpha|+|\beta|+1+n-2 m<0$, we take $p=0$, then:

$$
\left|D_{\xi}^{p} K_{r j}\left(\hat{\xi}, x_{1} t^{1 / 2 m}, \alpha+\beta\right)\right| \leqq M|\hat{\xi}|^{-n} \quad \text { for }|\hat{\xi}| \geqq 1 .
$$

Therefore:

$$
\left|D_{\xi}^{p} K_{r j}\left(\hat{\xi}, x_{1} t^{1 / 2 m}, \alpha+\beta\right)\right| \leqq M\left(1+|\hat{\xi}|^{n}\right)^{-1} \quad \text { for all } \hat{\xi} .
$$

We get:

$$
D^{\alpha+\beta} v\left(x_{1}, \hat{x}, t\right)=O(1) t^{-\varepsilon / 2 m}\left(1+|\hat{x}|^{N}\right)^{-1} \quad \text { for } x_{1} \geqq 0 .
$$

The lemma is proved.

THEOREM 3.2. Let $A$ be a positively strongly elliptic operator on $E^{n}$, homogeneous, of order $2 m$, with constant coefficients. Let $B_{1}, \cdots, B_{m}$ be $m$ homogeneous, differential operators on $E^{n}$ of order $r_{j}<2 m-1$ with constant coefficients. Let $t$ be a positive parameter such that $(A, \gamma)$ is formally positive. Then $G(x y, t)$ is given by the expression:

$$
G(x, y, t)=(2 \pi)^{-n} \int_{E^{n}} \exp (i\langle x-y, \xi\rangle)[a(\xi)+t]^{-1} d \xi+v(x, y, t)
$$

where the integral is taken as the Fourier transform of a tempered distribution if $2 m<n ; v(x, y, t)$ is given by Theorem 3.1.

(1) $G(x, y, t)$ is infinitely differentiable for $x \neq y$,

(2) $D^{\alpha} G(x, y, t)=O(1) t^{-\varepsilon / 2 m}|x-y|^{n+2 m-|\alpha|-\varepsilon}\left(1+|x-y|^{N}\right)^{-1}$ if $2 m-n \leqq|\alpha|$ and

$$
D^{\alpha} G(x, y, t)=O(1) t^{-\varepsilon / 2 m}\left(1+|x-y|^{N}\right)^{-1}, \quad \text { if } 0 \leqq|\alpha|<2 m-n,
$$

where $0<\varepsilon<1$ and $N$ is an arbitrary positive number.

Proof. The theorem follows immediately from Lemma 3.1 and Theorem 3.1.

TheOREM 3.3. Let $G(x, y, t)$ be the Green's function defined in Theorem 3.2 and $2 m>n$; then:

(1) $x \neq y, t^{1-n / 2 m} G(x, y, t) \rightarrow 0$ as $t \rightarrow+\infty$,

(2) $t^{1-n / 2 m} G(x, x, t) \sim(2 \pi)^{-n} \int_{E^{n}}[a(\xi)+1]^{-1} d \xi+O(1) t^{-\varepsilon / 2 m}$ as $t \rightarrow+\infty$.

Proof. Let $t=\tau^{2 m}$ and make the change of variables $\hat{\xi}=\tau \hat{\xi}^{\prime} ; \zeta_{1}=\tau \zeta_{1}^{\prime}$. We obtain:

$$
G(x, y, t)=\tau^{n-2 m} E(\tau x, \tau y, 1)+v(x, y, t) .
$$

We consider the expression $v(x, y, t)$. From Lemma 3.4, we have: 


$$
\begin{aligned}
v(x, y, t)=\sum_{r, j=1}^{m} \tau^{n-1-r_{j}} \int_{E^{n-1}} \exp (i\langle\tau \hat{x}-\tau \hat{y}, \hat{\xi}\rangle) Q_{r j}(\hat{\xi}, 1) H_{j}\left(\hat{\xi}, y_{1}, t\right) \\
\cdot \int_{C_{\xi}} \zeta_{1}^{r-1} \exp \left(i \zeta_{1} \tau x_{1}\right)\left[a\left(\zeta_{1}, \hat{\xi}\right)+1\right]^{-1} d \zeta_{1} d \hat{\xi} .
\end{aligned}
$$

On the other hand; $H_{j}\left(\hat{\xi}, y_{1}, t\right)=O(1) \tau^{r_{j}+1-2 m-\varepsilon}\left(1+|\hat{\xi}|^{2 m-r_{j}}\right)^{-1}$. When $x \neq y$ then by the Riemann-Lebesgue theorem, we have:

$$
\tau^{2 m-n} G(x, y, t) \rightarrow 0 \text { as } t \rightarrow+\infty .
$$

THEOREM 3.4. Let $G_{(p)}(x, y, t)$ be the pth iterate of the Green's function $G$ defined by Theorem 3.1. Let $p$ be such that $2 m p>n$; then:

(1) If $x \neq y, t^{p-n / 2 m} G_{(p)}(x, y, t) \rightarrow 0$ as $t \rightarrow+\infty$,

(2) $t^{p-n / 2 m} G_{(p)}(x, x, t)=(2 \pi)^{-n} \int_{E^{n}}[a(\xi)+1]^{-p} d \xi+O(1) t^{-\varepsilon / 2 m}, \quad 0<\varepsilon<1$, $t \rightarrow+\infty$.

Proof. We construct the iterates of $G$. We know that:

$$
G_{(2)}(x, y, t)=\int_{E^{n}} G(x, z, t) G(z, y, t) d z, \quad x \neq y .
$$

The integral is well defined. We also have:

$$
\begin{aligned}
F_{\hat{x}} G(x, z, t)= & (2 \pi)^{-(n-1) / 2} \exp (-i\langle\hat{z}, \hat{\xi}\rangle) \int_{E^{\prime}} \exp \left(i\left(x_{1}-z_{1}\right) \xi_{1}\right)\left[a\left(\xi_{1}, \hat{\xi}\right)+t\right]^{-1} d \xi_{1} \\
& +\exp (-i\langle\hat{z}, \hat{\xi}\rangle) V\left(x_{1}, z_{1}, \hat{\xi}, t\right)
\end{aligned}
$$

with:

$$
V\left(x_{1}, z_{1}, \hat{\xi}, t\right)=\sum_{r, j=1}^{m} Q_{r j}(\hat{\xi}, t) H_{j}\left(\hat{\xi}, z_{1}, t\right) \int_{c_{\xi, t}} \zeta_{1}^{r-1} \exp \left(i \zeta_{1} x_{1}\right)\left[a\left(\zeta_{1}, \hat{\xi}\right)+t\right]^{-1} d \zeta_{1}
$$

Consider the Fourier transform of $G_{(2)}(x, y, t)$ with respect to the tangential variables $\hat{x}$.

$$
\begin{aligned}
F_{\hat{x}} G_{(2)}(x, y, t) & =(2 \pi)^{(1-n) / 2} \int_{E^{n-1}} \exp (-i\langle\hat{x}, \hat{\xi}\rangle) G_{(2)}(x, y, t) d \hat{x}, \quad x \neq y, \\
& =(2 \pi)^{(1-n) / 2} \int_{E^{n-1}} \int_{E^{n}} \exp (-i\langle\hat{x}, \hat{\xi}\rangle) G(x, z, t) G(y, z, t) d z d \hat{x} .
\end{aligned}
$$

By Fubini's theorem we may interchange the order of integration. We obtain:

$$
\begin{gathered}
F_{\hat{x}} G_{(2)}(x, y, t)=\int_{E^{n}} F_{\hat{x}} G(x, z, t) G(y, z, t) d z, \\
F_{\hat{x}} G_{(2)}(x, y, t) \\
=(2 \pi)^{(1-n) / 2} \int_{E^{n}} \int_{E^{1}} \exp (-i\langle\hat{z}, \hat{\xi}\rangle) \exp \left(i \xi_{1}\left(x_{1}-z_{1}\right)\right)\left[a\left(\xi_{1}, \hat{\xi}\right)+t\right]^{-1} G(y, z, t) d \xi_{1} d z \\
+(2 \pi)^{(1-n) / 2} \int_{E^{n}} \exp (-i\langle\hat{z}, \hat{\xi}\rangle) V\left(\hat{\xi}, x_{1}, z_{1}, t\right) G(y, z, t) d z .
\end{gathered}
$$


From Theorem 3.2; Lemmas 3.2; 3.3; it follows that all the integrals are well defined. The first integral of the above expression may be written as follows: $(2 \pi)^{(1-n) / 2} \int_{E^{n}} \exp (-i\langle\hat{z}, \hat{\xi}\rangle) G(y, z, t) \int_{E^{1}} \exp \left[i \xi_{1}\left(x_{1}-z_{1}\right)\right]\left[a\left(\xi_{1}, \hat{\xi}\right)+t\right]^{-1} d \xi_{1} d z$

Denote by $k\left(z_{1}, x_{1}, t, \hat{\xi}\right)=\int_{E^{1}} \exp \left[i \xi_{1}\left(x_{1}-z_{1}\right)\right]\left[a\left(\xi_{1}, \hat{\xi}\right)+t\right]^{-1} d \xi_{1}$. Then:

$$
\begin{aligned}
& (2 \pi)^{(1-n) / 2} \int_{E^{n}} \exp (-i\langle\hat{z}, \hat{\xi}\rangle) G(y, z, t) k\left(z_{1}, x_{1}, \hat{\xi}, t\right) d z \\
& \quad=(2 \pi)^{(1-n) / 2} \int_{E^{n}} k\left(z_{1}, x_{1}, \hat{\xi}, t\right) \int_{E^{n-1}} \exp (-i\langle\hat{z}, \hat{\xi}\rangle) G(y, z, t) d \hat{z} d z_{1} \\
& \quad=\int_{E^{1}} k\left(z_{1}, x_{1}, \hat{\xi}, t\right) F_{2} G(x, y, t) d z_{1} \\
& =(2 \pi)^{(1-n) / 2} \int_{E^{1}} k\left(z_{1}-x_{1}, \hat{\xi}, t\right) \exp (-i\langle\hat{y}, \hat{\xi}\rangle) \int_{E^{1}} \exp \left[i \eta_{1}\left(z_{1}-y_{1}\right)\right]\left[a\left(\eta_{1}, \hat{\xi}\right)+t\right]^{-1} d \eta_{1} d z_{1} \\
& \quad+(2 \pi)^{(1-n) / 2} \int_{E^{1}} k\left(z_{1}-x_{1}, \hat{\xi}, t\right) \exp (-i\langle\hat{y}, \hat{\xi}\rangle) V\left(\hat{\xi}, y_{1}, z_{1}, t\right) d z_{1} .
\end{aligned}
$$

Consider the first term. It is easy to see that $k(u, \hat{\xi}, t)$ is integrable with respect to $u$. Applying Fubini's theorem, we get:

since

$$
(2 \pi)^{-n / 2} \exp (-i\langle\hat{y}, \hat{\xi}\rangle) \int_{E^{1}} \exp \left[i \xi_{1}\left(x_{1}-y_{1}\right)\right]\left[a\left(\xi_{1}, \hat{\xi}\right)+t\right]^{-2} d \xi_{1}
$$

$$
\int_{E^{1}} \exp \left(i \eta_{1} z_{1}\right) k\left(z_{1}-x_{1}, \hat{\xi}, t\right) d z_{1}=(2 \pi)^{-1 / 2} \exp \left(i \eta_{1} x_{1}\right)\left[a\left(\eta_{1}, \hat{\xi}\right)+t\right]^{-1}
$$

We consider:

$$
(2 \pi)^{(1-n) / 2} \int_{E^{n}} \exp (-i\langle\hat{z}, \hat{\xi}\rangle) V\left(\hat{\xi}, x_{1}, z_{1}, t\right) G(y, z, t) d z
$$

As before we may write it as

$$
\int_{E^{1}} V\left(\hat{\xi}, x_{1}, z_{1}, t\right)\left\{(2 \pi)^{(1-n) / 2} \int_{E^{n-1}} \exp (-i\langle\hat{z}, \hat{\xi}\rangle) G(y, z, t) d \hat{z}\right\} d z_{1} .
$$

The integral in the bracket is the Fourier transform of $G$ with respect to the tangential variable $\hat{z}$. We obtain:

$$
\begin{aligned}
& \int_{E^{1}} \int_{E^{1}} \exp (-i\langle\hat{y}, \hat{\xi}\rangle) \exp \left[i \xi_{1}\left(z_{1}-y_{1}\right)\right] V\left(\hat{\xi}, y_{1}, z_{1}, t\right)\left[a\left(\xi_{1}, \hat{\xi}\right)+t\right]^{-1} d \xi_{1} d z_{1} \\
& \quad+\int_{E^{1}} \exp (-i\langle\hat{y}, \hat{\xi}\rangle) V\left(\hat{\xi}, t, y_{1}, z_{1}\right) V\left(\hat{\xi}, t, y_{1}, x_{1}\right) d z_{1}
\end{aligned}
$$


Denote the first integral by $h_{1}\left(\hat{\xi}, x_{1}, y_{1}, t\right)$ and the second one by $h_{2}\left(\hat{\xi}, x_{1}, y_{1}, t\right)$; then:

$$
\begin{aligned}
F_{\hat{x}} G_{(2)}(x, y, t)= & (2 \pi)^{(1-n) / 2} \exp (-i\langle\hat{y}, \hat{\xi}\rangle) \int_{E^{1}} \exp \left[i \xi_{1}\left(x_{1}-y_{1}\right)\right]\left[a\left(\xi_{1}, \hat{\xi}\right)+t\right]^{-2} d \xi_{1} \\
& +h_{1}\left(\hat{\xi}, x_{1}, y_{1}, t\right)+h_{2}\left(\hat{\xi}, x_{1}, y_{1}, t\right)+g\left(\hat{\xi}, x_{1}, y_{1}, t\right)
\end{aligned}
$$

with

$$
g\left(\hat{\xi}, x_{1}, y_{1}, t\right)=\int_{E^{1}} \exp (-i\langle\hat{y}, \hat{\xi}\rangle) k\left(z_{1}-x_{1}, \hat{\xi}, t\right) V\left(\hat{\xi}, z_{1}, y_{1}, t\right) d z_{1} \cdot
$$

If $4 m>n$, we want to take the inverse Fourier transform of $F_{\dot{x}} G_{(2)}$. First we establish the following lemma:

LEMMA 3.5. Let $g\left(\hat{\xi}, x_{1}, y_{1}, t\right) ; h_{1}\left(\hat{\xi}, x_{1}, y_{1}, t\right), h_{2}\left(\hat{\xi}, x_{1}, y_{1}, t\right)$ be as above; then the following estimates hold uniformly for $t \geqq t_{0}>0$

(1) $g\left(\hat{\xi}, x_{1}, y_{1}, t\right)=O\left(\left(1+|\hat{\xi}|^{4 m}\right)^{-1}\right)$,

(2) $h_{1}\left(\hat{\xi}, x_{1}, y_{1}, t\right)=O\left(\left(1+|\xi|^{4 m}\right)^{-1}\right)$,

(3) $h_{2}\left(\hat{\xi}, x_{1}, y_{1}, t\right)=O\left(\left(1+|\hat{\xi}|^{4 m}\right)^{-1}\right)$.

Proof. Making the change of variable $\hat{\xi}=t^{1 / 2 m} \hat{\xi}^{\prime}$ and taking into account the results of Lemmas 3.2, 3.3, we get the above estimates immediately. We return to the proof of the theorem. If $4 m>n$, we may take the inverse Fourier transform of $F_{\hat{x}} G_{(2)}$ with respect to $\hat{\xi}$. We obtain:

$$
\begin{aligned}
G_{(2)}(x, y, t)= & (2 \pi)^{-n} \int_{E^{n}} \exp (-i\langle x-y, \xi\rangle)[a(\xi)+t]^{-2} d \xi \\
& +(2 \pi)^{(1-n) / 2} \int_{E^{n-1}} \exp (i\langle\hat{x}, \hat{\xi}\rangle)\left\{h_{1}\left(\hat{\xi}, x_{1}, y_{1}, t\right)+h_{2}\left(\xi, x_{1}, y_{1}, t\right)\right\} d \hat{\xi} .
\end{aligned}
$$

If $4 m<n$, we construct $F_{\hat{x}} G_{(3)}(x, y, t)$ etc. step by step. We take only the first term and we have to make an estimate of the error involved in terms of the parameter $t$, for large $t$.

From the proof of Theorem 3.3, we have:

$$
\begin{aligned}
& g\left(\hat{\xi}, x_{1}, y_{1}, t\right)=O(1) \tau^{-4 m+1-\varepsilon}\left(1+|\hat{\xi}|^{4 m-1}\right)^{-1}, \\
& h_{1}\left(\xi, x_{1}, y_{1}, t\right)=O(1) \tau^{-4 m+1-\varepsilon}\left(1+|\hat{\xi}|^{4 m-1}\right)^{-1}, \\
& h_{2}\left(\xi, x_{1}, y_{1}, t\right)=O(1) \tau^{-4 m+1-\varepsilon}\left(1+|\xi|^{4 m-1}\right)^{-1} .
\end{aligned}
$$

Therefore if $4 m>n$, we have:

$$
G_{(2)}(x, y, t)=(2 \pi)^{-n} \int_{E^{n}} \exp (i\langle x-y, \xi\rangle)[a(\xi)+t]^{-2} d \xi+O(1) t^{-2+(n-\varepsilon) / 2 m}
$$

More generally if $2 m p>n$,

$$
G_{(p)}(x, y, t)=(2 \pi)^{-n} \int_{E^{n}} \exp (i\langle x-y, \xi\rangle)[a(\xi)+t]^{-p} d \xi+O(1) t^{-p+(n-\varepsilon) / 2 m}
$$


The conclusion of the theorem follows immediately.

4. In this section the Green's function $\mathscr{G}(x, y, t)$ corresponding to the elliptic boundary value problem $\left\{A+t I, B_{j}, j=1, \cdots, m\right\}$ where $A$ and $B_{j}$ are defined respectively on $S$ and on $\Gamma$ and have infinitely differentiable coefficients is constructed. We will:

(1) Construct the Green's function $G$ associated with the elliptic boundary value problem $\left\{A+t I ; B_{j} ; j=1, \cdots, m\right\}$ where $A$ and $B_{j}$ are defined on $E_{+}^{n}, E^{n-1}$ respectively, with infinitely differentiable coefficients.

(2) Seek an integral representation of a function $u(x)$, infinitely differentiable function with compact support in $E_{+}^{n} \cup E^{n-1}$ in terms of $(A+t I) u, B_{j} u$.

(3) Get the function $\mathscr{G}$ using (1) and (2).

Lemma 4.1. Let $H_{j y}\left(x_{1}, \hat{x}-\hat{y}, t\right)$ be given by the expression:

$H_{j \hat{y}}\left(x_{1}, \hat{x}-\hat{y}, t\right)$

$=\sum_{r=1}^{m} \int_{E^{n-1}} \exp (i\langle\hat{x}-\hat{y}, \hat{\xi}\rangle) Q_{r j}(\hat{y}, \hat{\xi}, t) \int_{C_{\xi, t}} \zeta_{1}^{r-1} \exp \left(i \zeta_{1} x_{1}\right)\left[a\left(\hat{y}, \zeta_{1}, \hat{\xi}\right)+t\right]^{-1} d \zeta_{1} d \hat{\xi}$

where $a\left(\hat{y}, \zeta_{1}, \hat{\xi}\right)$ is the characteristic form of the homogeneous regularly elliptic operator $A_{\hat{y}}$ with coefficients evaluated at $\hat{y} ; C_{\hat{\xi}, t}$ is a closed curve in the $\zeta_{1}$ upper half plane surrounding the roots of $a\left(\hat{y}, \zeta_{1}, \hat{\xi}\right)+t=0$ for fixed $\hat{\xi}, t$.

$Q_{r j}(\hat{y}, \hat{\xi}, t)$ are the elements of the transpose of the inverse of the matrix:

$$
\left(c_{r j}(\hat{y}, \hat{\xi}, t)=\int_{c_{\xi, t}} \zeta_{1}^{r-1} b_{j}\left(\hat{y}, \zeta_{1}, \hat{\xi}\right)\left[a\left(\hat{y}, \zeta_{1}, \hat{\xi}\right)+t\right]^{-1} d \zeta_{1}\right) .
$$

$b_{j}(\hat{y}, \xi)$ is the characteristic form of the differential operator $B_{j \hat{y}}$ of order $r_{j}$ and with coefficients evaluated at $\hat{y}$.

$H_{j y}\left(x_{1}, \hat{x}-\hat{y}, t\right)$ is infinitely differentiable for $\hat{x} \neq \hat{y}$ and:

$$
\begin{aligned}
\left(B_{k}-B_{k \hat{\jmath}}\right) H_{j \hat{y}}(0, \hat{x}-\hat{y}, t) & =O(1) t^{-\varepsilon / 2 m}|\hat{x}-\hat{y}|^{-n+2-\varepsilon}, \quad k, j=1, \cdots, m, \\
A H_{j \mathcal{\vartheta}}\left(x_{1}, \hat{x}-\hat{y}, t\right) & =O(1) t^{-\varepsilon / 2 m}|\hat{x}-\hat{y}|^{-n+2-\varepsilon}, \quad x_{1}>0 .
\end{aligned}
$$

Proof. $H_{j \hat{y}}\left(x_{1}, \hat{x}-\hat{y}, t\right)$ is well defined and for $x_{1}>0$ is infinitely differentiable (Lemmas 3.2, 3.3). We study the case when $x_{1} \rightarrow 0$. Let $p$ be a positive integer and consider:

$$
\begin{aligned}
(\hat{x}-\hat{y})^{p} \hat{D}^{\alpha} D_{1}^{\beta} H_{j \mathcal{P}}\left(x_{1}, \hat{x}-\hat{y}, t\right) & \\
= & \sum_{r=1}^{m} \int_{E^{n-1}} \exp (i\langle\hat{x}-\hat{y}, \hat{\xi}\rangle) \\
& \cdot D_{\xi}^{p}\left\{\hat{\xi}^{\alpha} Q_{r j}(\hat{\xi}, \hat{y}, t) \int_{C_{\xi, t}} \zeta_{1}^{r-1+\beta} \exp \left(i \zeta_{1} x_{1}\right)\left[a\left(\hat{y}, \zeta_{1}, \hat{\xi}\right)+t\right]^{-1} d \zeta_{1}\right\} d \hat{\xi} .
\end{aligned}
$$


The expression $D_{\xi}^{p}\{\}$ is integrable at the origin when $x_{1} \rightarrow 0$. We have only to consider it at infinity.

$$
\begin{aligned}
& D_{\xi}^{p}\left\{\hat{\xi}^{\alpha} Q_{r j}(\hat{y}, \hat{\xi}, t) \int_{C_{\xi, t}} \zeta_{1}^{\beta+r-1}\left[a\left(\hat{y}, \zeta_{1}, \hat{\xi}\right)+t\right]^{-1} d \zeta_{1}\right\} \\
& =t^{\left(|\alpha|+|\beta|-r_{j}-p\right) / 2 m} D_{\xi}^{p}\left\{\hat{\xi}^{\alpha} Q_{r j}(\hat{y}, \hat{\xi}, 1) \int_{C_{\xi, 1}} \zeta_{1}^{r-1+\beta}\left[a\left(\hat{y}, \zeta_{1}, \hat{\xi}\right)+1\right]^{-1} d \zeta_{1}\right\} .
\end{aligned}
$$

Making the change of variables $\hat{\xi}=\tau \hat{\xi}^{\prime}, \zeta_{1}=\tau \zeta_{1}^{\prime}$ with $\tau=|\hat{\xi}|$; we get:

$$
\begin{aligned}
& D_{\xi}^{p}\left\{\hat{\xi}^{\alpha} Q_{r j}(\hat{y}, \hat{\xi}, 1) \int_{C_{\xi, 1}} \zeta_{1}^{r-1+\beta}\left[a\left(\hat{y}, \zeta_{1}, \hat{\xi}\right)+1\right]^{-1} d \zeta_{1}\right\} \\
& \tau^{\alpha+\beta-r_{j}-p} D_{\xi^{\prime}}^{p}\left\{\hat{\xi}^{\prime \alpha} Q_{r j}\left(\hat{y}, \hat{\xi}^{\prime}, \tau^{-2 m}\right) \int_{C_{\xi^{\prime}}} \zeta_{1}^{r-1+\beta}\left[a\left(\hat{y}, \zeta_{1}, \hat{\xi}^{\prime}\right)+\tau^{-2 m}\right]^{-1} d \zeta_{1}\right\} .
\end{aligned}
$$

So:

$$
\begin{aligned}
D_{\xi}^{p} \mid \hat{\xi}^{\alpha} Q_{r j}(\hat{y}, \hat{\xi}, t) \int_{C_{\xi, t}} \zeta_{1}^{r-1+\beta}\left[a\left(\hat{y}, \zeta_{1}, \hat{\xi}\right)+t\right]^{-1} d \zeta_{1} \\
\quad=t^{\left(\alpha+\beta-r_{j}-p\right) / 2 m} O(1)|\hat{\xi}|^{-r_{j}-p+|\alpha|+|\beta|} \text { for large }|\hat{\xi}| .
\end{aligned}
$$

If we take $p=-r_{j}+n+|\alpha|+|\beta| \geqq 0$, the expression $(\hat{x}-\hat{y})^{p} \hat{D}^{\alpha} D_{1}^{\beta} H_{j \emptyset}$ is well defined. When $\hat{x}=\hat{y}$, it is equal to zero. We may replace $\exp (i\langle\hat{x}-\hat{y}, \hat{\xi}\rangle)$ by $\exp (i\langle\hat{x}-\hat{y}, \hat{\xi}\rangle)-1$ which is less than $|\hat{x}-\hat{y}|^{1-\varepsilon}|\hat{\xi}|^{-\varepsilon+1}$. We get:

$$
D^{\alpha} H_{j y}(0, \hat{x}-\hat{y}, t)=O(1) t^{-\varepsilon / 2 m}|\hat{x}-\hat{y}|^{r_{j}-n-|\alpha|-|\beta|+1-\varepsilon} .
$$

It follows that if $r_{k} \leqq r_{j}$ :

$$
\left(B_{k}-B_{k \hat{\jmath}}\right) H_{j \hat{y}}(0, \hat{x}-\hat{y}, t)=O(1) t^{-\varepsilon / 2 m}|\hat{x}-\hat{y}|^{-n+2-\varepsilon} .
$$

When $r_{k}>r_{j}$; consider

$$
\begin{aligned}
B_{k} H_{j j}\left(x_{1}, \hat{x}-\hat{y}, t\right)=\sum_{r=1}^{m} & \int_{E^{n-1}} \exp (i\langle\hat{x}-\hat{y}, \hat{\xi}\rangle) Q_{r j}(\hat{y}, \hat{\xi}, t) \\
& \cdot \int_{C_{\xi, t}} \zeta_{1}^{r-1} \exp \left(i \zeta_{1} x_{1}\right) b_{k}\left(\hat{x}, \zeta_{1}, \hat{\xi}\right)\left[a\left(\hat{y}, \zeta_{1}, \hat{\xi}\right)+t\right]^{-1} d \zeta_{1} d \hat{\xi} .
\end{aligned}
$$

It is well defined for $x_{1}>0$, has a discontinuity at $\left(x_{1}, \hat{x}-\hat{y}\right)=0$ and:

$$
\left.B_{k} H_{j y}\left(x_{1}, 0, t\right)\right|_{x_{1}=0}=0 .
$$

The integrand is nonnull for $\hat{x}=\hat{y} ; x_{1}>0$.

Consider: $B_{k} H_{j y}(|\hat{x}-\hat{y}|, \hat{x}-\hat{y}, t)$. We have:

$$
\left.B_{k} H_{j \vartheta}(|\hat{x}-\hat{y}|, \hat{x}-\hat{y}, t)\right|_{\hat{\imath}=0}=0 .
$$


$b_{k}\left(\hat{y}, \zeta_{1}, \hat{\xi}\right)$ is infinitely differentiable; taking the Taylor's development of $b_{k}$ in powers of $\hat{x}-\hat{y}$ for $\hat{x}$ near $\hat{y}$, up to the order $n+r_{k}+2$ and putting in the above integral, we obtain:

$$
\begin{aligned}
B_{k} H_{j y}\left(x_{1}, \hat{x}-\hat{y}, t\right)= & B_{k \hat{y}} H_{j y}\left(x_{1}, \hat{x}-\hat{y}, t\right)+\int_{E^{n-1}} \exp (i\langle\hat{x}-\hat{y}, \hat{\xi}\rangle) P_{j k}^{1}\left(\hat{\xi}, \hat{y}, x_{1}, t\right) d \hat{\xi} \\
& +\int_{E^{n-1}} \exp (i\langle\hat{x}-\hat{y}, \hat{\xi}\rangle) P_{j k}^{2}(\hat{\xi}, x, t, \hat{y}) d \hat{\xi} .
\end{aligned}
$$

The integrals are well defined for $x_{1}>0$ and the last expression is majorized by $M t^{-\varepsilon / 2 m}|\hat{x}-\hat{y}|^{-\varepsilon+r_{j}+1} \quad$ for $x_{1} \geqq 0$. The expression $P_{j k}^{1}\left(\xi, x_{1}, \hat{y}, t\right)$ is not integrable when $x_{1}=0$. Since $\left.B_{k} H_{j \xi}(|\hat{x}-\hat{y}|, \hat{x}-\hat{y}, t)\right|_{\hat{x}=\vartheta}=0$ we must have:

$$
P_{j k}^{1}(\hat{\xi}, 0, \hat{y}, t)=0 \text {. }
$$

It follows then that:

$$
\left(B_{k}-B_{k \vartheta}\right) H_{j \vartheta}\left(x_{1}, \hat{x}-\hat{y}, t\right)=O(1) t^{-\varepsilon / 2 m}|\hat{x}-\hat{y}|^{-n+2-\varepsilon}
$$

for all $j, k$.

We note that $(\hat{x}-\hat{y})^{n-2} A H_{j y}\left(x_{1}, \hat{x}-\hat{y}, t\right)$ is uniformly continuous in $\hat{x}-\hat{y}$ for $x_{1}>0$ and is equal to zero for $\hat{x}=\hat{y}$. So for large $t$ when $|\hat{x}-\hat{y}| \leqq t^{-\varepsilon / 2(2 m+2)}$, we have:

$$
\left|(\hat{x}-\hat{y})^{n-2} A H_{j \vartheta}\left(x_{1}, \hat{x}-\hat{y}, t\right)\right| \leqq M t^{-s}
$$

for some positive number $s$.

On the other hand, we have:

$$
\left|(\hat{x}-\hat{y})^{n+2 m-1+\varepsilon} A H_{j y}\left(x_{1}, \hat{x}-\hat{y}, t\right)\right| \leqq M t^{-\varepsilon / 2 m} .
$$

So for $|\hat{x}-\hat{y}|>t^{-\varepsilon / 2(2 m+2)}$, we get:

$$
\left|(\hat{x}-\hat{y})^{n-2} A H_{j g}\left(x_{1}, \hat{x}-\hat{y}, t\right)\right| \leqq M t^{-\varepsilon / 4 m} .
$$

Hence for large $t$ and $x_{1}>0$, we obtain:

$$
(\hat{x}-\hat{y})^{n-2} A H_{j 0}\left(x_{1}, \hat{x}-\hat{y}, t\right)=O(1) t^{-v / 2 m}, \quad 0<v<1 .
$$

LEMMA 4.2. Let $H_{j \vartheta}$ be as above, then:

$$
\begin{aligned}
& B_{k \vartheta} H_{j \ell}(0, \hat{x}-\hat{y}, t)=0 \quad \text { if } k \neq j, \\
& B_{j \vartheta} H_{j \vartheta}(0, \hat{x}-\hat{y}, t)=\delta_{\mathfrak{l}} .
\end{aligned}
$$

Proof. It follows immediately from the definition of $\boldsymbol{H}_{j}$.

LEMMA 4.3. Let $\left\{A ; B_{j} ; j=1, \cdots, m\right\}$ be a uniformly regularly elliptic boundary value problem where $A$ and $B_{j}$ are defined on $E_{+}^{n}, E^{n-1}$, have infinitely differentiable coefficients and are homogeneous of orders $2 m, r_{j}$ re- 
spectively. Let $A_{z}, B_{j z}$ be the operators obtained from $A ; B_{j}$ by taking the values of the coefficients at the point $z$. Let $G_{(z)}(x, z, t)$ be the Green's function associated with $\left\{A_{z}+t I ; B_{j z} ; j=1, \cdots, m\right\}$ constructed in $\S 3$, (Theorem 3.2).

Let

(1)

$$
\begin{aligned}
& \alpha_{0}(x, z, t)=\left(A-A_{z}\right) G_{(z)}(x, z, t), \quad x \neq z ; x, z \text { in } B^{+}, \\
& \alpha_{j}(x, z, t)=\left(B_{j}-B_{j z}\right) G_{(z)}(x, z, t), \quad j=1, \cdots, m, \\
& \alpha(x, z, t)=\left(\alpha_{0}, \cdots, \alpha_{m}\right), \\
& \beta_{0 k}(x, \hat{y}, t)=\left(A-A_{\hat{\jmath}}\right) H_{k \hat{\jmath}}(x, \hat{y}, t) \text {, }
\end{aligned}
$$

$$
\begin{aligned}
\beta_{j k}(\hat{x}, \hat{y}, t) & =\left(B_{j}-B_{j \mathfrak{j}}\right) H_{k \hat{\jmath}}(\hat{x}, \hat{y}, t), \quad j=1, \cdots, m, \\
\beta_{k}(x, \hat{y}, t) & =\left(\beta_{0 k}, \cdots, \beta_{m k}\right),
\end{aligned}
$$

$H_{k y}$ is given by Theorem 4.1.

$$
w(x, z, t)=\left(v(x, z), h(\hat{x}, \hat{z}), \cdots, h_{m}(\hat{x}, z)\right) .
$$

Define the linear transformations:

$$
\begin{aligned}
& T_{0} w(x, z, t)=\int_{B^{+}} \alpha(x, y, t) v(y, z) d y, \\
& T_{k} w(x, z, t)=\int_{\Gamma_{0}} \beta_{k}(x, \hat{y}, t) h_{k}(\hat{y}, \hat{z}) d \hat{y},
\end{aligned}
$$

with:

$$
\begin{gathered}
T w=T_{0} w+\sum_{k=1}^{m}\left(T_{k} w\right) \\
B^{+}=\left\{x:|x|<1, x_{1}>0\right\} ; \quad \Gamma_{0}=\left\{x_{i}|x|<1, x_{1}=0\right\} .
\end{gathered}
$$

Then the integral equation:

$$
w(x, z, t)+T w(x, z, t)+\alpha(x, z, t)=0
$$

may be solved by the Neumann series for sufficiently large $t$ and:

$$
\begin{aligned}
v(x, z, t) & =O(1) t^{-\varepsilon / 2 m}|x-z|^{1-n-\varepsilon}\left(1+\left|t^{1 / 2 m}(x-z)\right|^{N}\right)^{-1}, \\
h_{j}(\hat{x}, \hat{z}, t) & =O(1) t^{-\varepsilon / 2 m}|\hat{x}-\hat{z}|^{-n+2-\varepsilon}\left(1+\left|t^{1 / 2 m}(\hat{x}-\hat{z})\right|^{N}\right)^{-1},
\end{aligned}
$$

$t=\tau^{2 m}<\varepsilon<1 ; N$ any positive integer.

Proof. We have from Theorem 3.2:

$$
\begin{aligned}
& \alpha_{0}(x, z, t)=\left(A-A_{z}\right) G_{(z)}(x, z, t)=O(1) \tau^{-\varepsilon}|x-z|^{1-n-8}\left(1+|(x-z)|^{N}\right)^{-1}, \\
& \alpha_{j}(\hat{x}, z, t)=\left(B_{j}-B_{j z}\right) G_{(z)}(\hat{x}, z, t)=O(1) \tau^{-\varepsilon}|\hat{x}-\hat{z}|^{-n+2-8}\left(1+|(\hat{x}-\hat{z})|^{N}\right)^{-1} .
\end{aligned}
$$


Finally from Lemma 4.1:

$$
\beta_{j k}(\hat{x}, \hat{y}, t)=O(1) \tau^{-\varepsilon}|\hat{x}-\hat{y}|^{-n+2-\varepsilon} .
$$

Consider the series:

$$
w(z, x, t)=\alpha(x, z, t)+T \alpha+T^{2} \alpha+\cdots .
$$

It may be written as:

$$
\begin{aligned}
& v(x, z, t)=\alpha_{0}(x, z, t)+\int_{B^{+}} \alpha_{0}(x, y, t) \alpha_{0}(y, z, t) d y \\
& +\sum_{k=1}^{m} \int_{\Gamma_{0}} \beta_{0 k}(x, \hat{y}, t) \alpha_{k}(\hat{y}, \hat{z}) h_{j}(\hat{x}, z, t)=\alpha_{j}(\hat{x}, \hat{z}, t)+\int_{B^{+}} \alpha_{0}(\hat{x}, y, t) \alpha_{j}(y, \hat{z}, t) d y \\
& \quad+\sum_{k=1}^{m} \int_{\Gamma_{0}} \beta_{j k}(\hat{x}, \hat{y}, t) \alpha_{k}(\hat{y}, \hat{z}) d \hat{y}+\cdots, \quad j=1, \cdots, m .
\end{aligned}
$$

The first series is majorized by:

$$
\begin{aligned}
& O(1) t^{-\varepsilon / 2 m}|x-z|^{1-n-\varepsilon}+O(1) t^{-\varepsilon / 2 m} \int_{B^{+}}|x-y|^{1-n-\varepsilon}|y-z|^{1-n-\varepsilon} d y \\
& \quad+O(1) t^{-\varepsilon / m} \int_{\Gamma_{0}}|\hat{x}-\hat{y}|^{-n+2-\varepsilon}|\hat{z}-\hat{y}|^{-n+2-\varepsilon} d \hat{y}+\cdots,
\end{aligned}
$$

which is uniformly convergent for large $t ; x, z$ in $B^{+}$. The second series is majorized by:

$$
\begin{array}{r}
O(1) t^{-\varepsilon / 2 m}|\hat{x}-\hat{z}|^{2-n-\varepsilon}+O(1) t^{-\varepsilon / m} \int_{B^{+}}|x-y|^{1-n-\varepsilon}|y-z|^{1-n-\varepsilon} d y \\
+O(1) t^{-\varepsilon / m} \int_{\Gamma_{0}}|\hat{x}-\hat{y}|^{-n+2-\varepsilon}|\hat{z}-\hat{y}|^{2-n-\varepsilon} d \hat{y}+\cdots
\end{array}
$$

which is also uniformly convergent for large $t$.

The proof of the theorem is completed.

THEOREM 4.1. Let $\left\{A ; B_{j} ; j=1, \cdots, m\right\}$ be a uniformly regularly elliptic boundary value problem where $A, B_{j}$ are defined on $E_{+}^{n}, E^{n-1}$ and have infinitely differentiable coefficients. If $G_{(z)}(x, z, t)$ is the Green's function associated with the constant coefficients problem $\left\{A_{z}+t I ; B_{j z} ; j=1, \cdots, m\right\}$ constructed in Theorem 3.2; then:

$$
G(x, z, t)=G_{(z)}(x, z, t)+\int_{B^{+}} G_{(y)}(x, y, t) v(y, z, t) d y+\sum_{k=1}^{m} \int_{\Gamma_{0}} H_{k \rho}\left(x_{1}, \hat{y}, t\right) h_{k}(\hat{y}, z)
$$

is the Green's function associated with the elliptic boundary value problem $\left\{A+t I ; B_{j} ; j=1, \cdots, m\right\} . H_{k \vartheta}$ are the kernels defined by Lemmas 4.1, $v, h_{k}$ are the solutions of the system of integral equations of Lemma 4.3. 
1966] ASYMPTOTIC DISTRIBUTION OF EIGENVALUES AND EIGENFUNCTIONS 535

$$
B^{+}=\left\{x: x_{1}>0 ;|x|<1\right\} ; \quad \Gamma_{0}=\left\{x: x_{1}=0 ;|x|<1\right\} .
$$

Proof. We verify that

$$
\begin{aligned}
(A+t I) G(x, z, t) & =\delta_{z}, \\
B_{j} G(x, z, t) & =0, \quad x_{1}=0 ; j=1, \cdots, m .
\end{aligned}
$$

(1) Consider $(A+t I) G(x, z, t)$. We have:

$$
\begin{aligned}
(A+t I) G(x, z, t)= & \left(A_{z}+t I\right) G_{(z)}(x, z, t)+\left(A-A_{z}\right) G_{z}(x, z, t) \\
& +\sum_{k=1}^{m} \int_{\Gamma_{0}}\left(A-A_{\hat{\jmath}}\right) H_{k \mathcal{\jmath}}(x, \hat{y}, t) h_{k}(\hat{y}, t, z) d \hat{y} \\
& +(A+t I)\left(\int_{B^{+}} G_{y}(x, y, t) v(y, z, t) d y\right) .
\end{aligned}
$$

(2) Let $\phi(x)$ be an infinitely differentiable function with compact support in $B^{+}$. We have:

$$
\begin{aligned}
\int_{B^{+}}(A+t I) G(x, z, t) \phi(x) d x= & \phi(z)+\int_{B^{+}}\left(A-A_{z}\right) G_{z}(x, t, z) \phi(x) d x \\
& +\sum_{k=1}^{m} \int_{B^{+}}\left(A-A_{\hat{y}}\right) H_{k \mathcal{\jmath}}(x, \hat{y}, t) h_{k}(\hat{y}, z, t) \phi(x) d \hat{y} d x \\
& +\int_{B^{+}}(A+t I)\left(\int_{B^{+}} G_{y}(x, y, t) v(y, z, t) d y\right) \phi(x) d x
\end{aligned}
$$

(3) Consider the last integral. Since $\phi(x) \in C_{c}^{\infty}\left(B^{+}\right)$we may write it as:

$$
\begin{aligned}
\sum_{|\alpha|=2 m} & \int_{B^{+}} \int_{B^{+}} G_{y}(x, t, y) v(y, z, t)\left[t \phi+D_{x}^{\alpha}\left(\tilde{a}_{\alpha}(x) \phi(x)\right)\right] d y d x \\
& =\sum_{|\alpha|=2 m} \int_{B^{+}} \int_{B^{+}}\left[t \phi(x)+D_{x}^{\alpha}\left(\tilde{a}_{\alpha}(x) \phi(x)\right)\right] G_{y}(x, y, t) v(y, z, t) d x d y
\end{aligned}
$$

by Fubini's theorem. Integrating by parts, we obtain:

$$
\begin{aligned}
\int_{B^{+}} \int_{B^{+}}(A+t I) G_{y}(x, y, t) v(y, z, t) \phi(x) d x d y \\
=\int_{B^{+}} \int_{B^{+}}\left(A_{y}+t I\right) G_{y}(x, y, t) \phi(x) v(y, z, t) d x d y \\
\quad+\int_{B^{+}} \int_{B^{+}}\left(A-A_{y}\right) G_{y}(x, y, t) v(y, z) \phi(x) d x d y \\
=\int_{B^{+}} \phi(y) v(y, z, t) d y+\int_{B^{+}} \int_{B^{+}}\left(A-A_{y}\right) G_{y}(x, y, t) v(y, z, t) \phi(x) d y d x
\end{aligned}
$$


by Fubini's theorem.

(4) But $v, h_{j}$ satisfy the equation:

$$
\begin{aligned}
0= & v(x, z, t)+\left(A-A_{z}\right) G_{z}(x, z, t)+\int_{B^{+}}\left(A-A_{y}\right) G_{y}(x, y, t) v(y, z) d y \\
& +\sum_{k=1}^{m} \int_{\Gamma_{0}}\left(A-A_{\hat{y}}\right) H_{k \hat{\jmath}}(x, \hat{y}, t) h_{k}(\hat{y}, z, t) d \hat{y} .
\end{aligned}
$$

Hence: $(A+t I) G(x, z, t)=\delta_{z}$.

(5) We show that $B_{j} G(x, z, t)=0$ if $x_{1}=0 ; j=1, \cdots, m$. We have with $x, z$ in $B^{+}$:

$$
\begin{aligned}
B_{j} G(x, z, t)= & B_{j z} G_{z}(x, z, t)+\left(B_{j}-B_{j z}\right) G_{z}(x, z, t)+\int_{B^{+}} B_{j} G_{y}(x, y, t) v(y, z) d y \\
& +\sum_{k=1}^{m} \int_{\Gamma_{0}} B_{j} H_{k \vartheta}(x, \hat{y}, t) h_{k}(\hat{y}, z, t) d \hat{y} .
\end{aligned}
$$

The differentiations under the integral sign are valid (Theorems 3.2, 4.1). The last integral may be written as:

$$
\int_{\Gamma_{0}}\left(B_{j}-B_{j \hat{y}}\right) H_{k \hat{\vartheta}}(x, \hat{y}, t) h_{k}(\hat{y}, z, t) d \hat{y}+\int_{\Gamma_{0}} B_{j \mathcal{y}} H_{k \hat{y}}(x, \hat{y}, t) h_{k}(\hat{y}, z, t) d \hat{y}
$$

Let $\phi(\hat{x}) \in C_{c}^{\infty}\left(\Gamma_{0}\right)$. Consider:

$$
\int_{\Gamma_{0}} \int_{\Gamma_{0}} B_{j \hat{\vartheta}} H_{k \hat{y}}(x, \hat{y}, t) h_{k}(\hat{y}, z, t) \phi(\hat{x}) d \hat{x} d \hat{y} .
$$

By Fubini's theorem, we have:

$$
\int_{\Gamma_{0}} \int_{\Gamma_{0}} B_{j \ell} H_{k \hat{\rho}}(x, \hat{y}, t) h_{k}(\hat{y}, z, t) \phi(\hat{x}) d \hat{x} d \hat{y}
$$

We know that $B_{j \hat{y}} H_{k \hat{y}}(\hat{x}, \hat{y})=\delta_{j k} \delta_{\hat{y}}$ so as $x_{1} \rightarrow 0$, we get:

$$
\delta_{j k} \int_{\Gamma_{0}} h_{k}(\hat{y}, z, t) \phi(\hat{y}) d \hat{y} .
$$

(6) On the other hand, $v$ and $h_{k}$ satisfy the equation:

$$
\begin{aligned}
\left(B_{j}-B_{j \mathfrak{z}}\right) G_{z}(\hat{x}, \hat{z}, t) & +\int_{B^{+}}\left(B_{j}-B_{j \mathcal{g}}\right) G_{y}(\hat{x}, \hat{y}, t) v(\hat{y}, z, t) d \hat{y} \\
& +\sum_{k=1}^{m} \int_{\Gamma_{0}} B_{j y} H_{k \hat{y}}(\hat{x}, \hat{y}, t) h_{k}(\hat{y}, z, t) d \hat{y}+h_{j}(z, x, t)=0 ; \quad j=1, \cdots, m .
\end{aligned}
$$

So $B_{j} G(\hat{x}, z, t)=0, j=1, \cdots, m$. 
LEMma 4.4. Let $\left\{A ; B_{j} ; j=1, \cdots, m\right\}$ be a uniformly regularly elliptic boundary value problem where $A$ and $B_{j}$ are defined on $E_{+}^{n}, E^{n-1}$ and have infinitely differentiable coefficients. $A$ and $B_{j}$ are homogeneous differential operators. Let $G_{z}(x, z, t)$ be the Green's function corresponding to the constant coefficients problem $\left\{A_{z}+t I ; B_{j z} ; j=1, \cdots, m\right\} . G_{z}$ is given by Theorem 3.2.

Let $H_{j \hat{y}}(x, \hat{y})$ be the kernels given in Lemma 4.1. Set:

$$
\begin{aligned}
\alpha_{0 j}(x, \hat{y}) & =\left(A-A_{\hat{\vartheta}}\right) H_{j y}(x, \hat{y}), \\
\alpha_{r j}(\hat{x}, \hat{y}) & =\left(B_{r}-B_{r \vartheta}\right) H_{j \jmath}(\hat{x}, \hat{y}), \\
\alpha_{j}(x, \hat{y}) & =\left(\alpha_{0 j}, \cdots, \alpha_{m j}\right), \\
\beta_{0}(x, z) & =\left(A-A_{z}\right) G_{z}(x, z), \\
\beta_{r}(\hat{x}, z) & =\left(B_{r}-B_{r z}\right) G_{z}(x, z), \\
\beta(x, z) & =\left(\beta_{0}, \cdots, \beta_{m}\right), \\
w_{j}(x, \hat{y}) & =\left(v_{j}, h_{j}, \cdots, h_{m j}\right) .
\end{aligned}
$$

Define the transformations:

$$
\begin{aligned}
T w_{j}(x, \hat{y}) & =\int_{B^{+}} \beta(x, z) v_{j}(z, \hat{y}) d z, \\
T_{k} w_{j}(x, \hat{y}) & =\int_{\Gamma_{0}} \alpha_{k}(x, z) h_{j k}(z, \hat{y}) d z, \\
T w_{j}+\sum_{k=1}^{m} T_{k} w_{j} & =\mathscr{I} w_{j}(x, \hat{y}) .
\end{aligned}
$$

Then the integral equation:

$$
w_{j}(x, y)+\mathscr{I} w_{j}(x, y)+\alpha_{j}(x, y)=0, \quad j=1, \cdots, m,
$$

may be solved by the Neumann series for large $t$ and for $x, y$ in $B^{+} \cup \Gamma=\left\{x:|x|<1 ; x_{1} \geqq 0\right\}$.

Moreover:

$$
\begin{aligned}
& v_{j}(x, \hat{y})=O(1) t^{-\varepsilon / 2 m}|\hat{x}-\hat{y}|^{-n+2-\varepsilon}\left(1+|(x-\hat{y})|^{N}\right)^{-1}, \\
& h_{j k}(\hat{x}, \hat{y})=O(1) t^{-\varepsilon / 2 m}|\hat{x}-\hat{y}|^{-n+2-\varepsilon}\left(1+|(\hat{x}-\hat{y})|^{N}\right)^{-1},
\end{aligned}
$$

$0<\varepsilon<1 ; N$ is a positive integer.

Proof. From Lemma 4.1, we have:

$$
\begin{aligned}
& \alpha_{0 j}(x, \hat{y})=\left(A-A_{\hat{\jmath}}\right) H_{j,}(x, \hat{y})=O(1) t^{-\varepsilon / 2 m}|x-\hat{y}|^{-n+2-\varepsilon}, \\
& \alpha_{r j}(\hat{x}, \hat{y})=\left(B_{r}-B_{r \hat{\jmath}}\right) H_{j,}(\hat{x}, \hat{y})=O(1) t^{-\varepsilon / 2 m}|\hat{x}-\hat{y}|^{-n+2-\varepsilon} .
\end{aligned}
$$

From Theorem 3.2, we get: 


$$
\begin{aligned}
& \beta_{0}(x, z)=\left(A-A_{z}\right) G_{z}(x, z)=O(1) t^{-\varepsilon / 2 m}|x-z|^{-n+1-\varepsilon}, \\
& \beta_{r}(\hat{x}, z)=\left(B_{r}-B_{r z}\right) G_{z}(\hat{x}, z)=O(1) t^{-\varepsilon / 2 m}|\hat{x}-\hat{z}|^{-n+2-\varepsilon} .
\end{aligned}
$$

Consider the series:

$$
\alpha_{j}(x, \hat{y})+\mathscr{I} \alpha_{j}(x, \hat{y})+\mathscr{I} \alpha_{j}+\cdots .
$$

It may be written as:

and:

$$
\alpha_{0 j}(x, \hat{y})+\int_{B^{+}} \beta_{0}(x, z, t) \alpha_{0 j}(z, \hat{y}) d z+\sum_{k=1}^{m} \int_{\Gamma_{0}} \alpha_{0_{k}}(x, \hat{z}) \alpha_{j k}(\hat{z}, \hat{y}) d \hat{z}+\cdots
$$

$$
\alpha_{r j}(\hat{x}, \hat{y})+\int_{B^{+}} \beta_{r}(\hat{x}, z, t) \alpha_{0 j}(z, \hat{y}) d z+\sum_{k=1}^{m} \int_{\Gamma_{0}} \alpha_{r k}(\hat{x}, \hat{z}) \alpha_{j k}(\hat{z}, \hat{y}) d \hat{z}+\cdots .
$$

They are majorized by the series:

$$
\begin{gathered}
O(1) t^{-\varepsilon / 2 m}|\hat{x}-\hat{y}|^{-n+2-\varepsilon}+O(1) t^{-\varepsilon / m} \int_{B^{+}}|\hat{x}-\hat{z}|^{-n+2-\varepsilon}|\hat{y}-\hat{z}|^{-n+2-\varepsilon} d z \\
+O(1) t^{-\varepsilon / m} \int_{\Gamma_{0}}|\hat{x}-\hat{z}|^{-n+2-\varepsilon}|\hat{z}-\hat{y}|^{-n+2-\varepsilon} d \hat{z}+\cdots
\end{gathered}
$$

which is uniformly convergent for large $t$.

The lemma is proved.

LEMMA 4.5. Let $H_{j \mathbf{z}}(x, \hat{z})$ be the kernels constructed in Lemma 4.1 for the constant coefficients problem $\left\{A_{2}+t I ; B_{j 2} ; j=1, \cdots, m\right\}$ Let $G_{z}(x, z, t)$ be the Green's function associated with the elliptic boundary value problem $\left\{A_{z}+t I ; B_{j z} ; j=1, \cdots, m\right\}$. The differential operators $A, B_{j}$ are homogeneous and have infinitely differentiable coefficients.

Let:

$$
H_{j}(x, \hat{y})=H_{j \hat{y}}(x, \hat{y})+\int_{B^{+}} G_{z}(x, z, t) v_{j}(z, y) d z+\sum_{k=1}^{m} \int_{\Gamma_{0}} H_{k \hat{z}}(x, \hat{z}) h_{k j}(\hat{z}, \hat{y}) d \hat{z}
$$

where $v_{j}$ and $h_{k j}$ satisfy the system of integral equations of Lemma 4.4. Then:

$$
\begin{aligned}
(A+t I) H_{j}(x, \hat{y}) & =0, \quad x \text { in } B^{+}=\left\{x: x_{1}>0,|x|<1\right\}, \\
B_{r} H_{r}(0, \hat{x}, \hat{y}) & =\delta_{\hat{y}} \\
B_{r} H_{j}(0, \hat{x}, \hat{y}) & =0 \text { if } r \neq j .
\end{aligned}
$$

Proof. The proof is long but easy and is similar to that of Theorem 4.2.

THEOREM 4.2. Let $u(x)$ be an infinitely differentiable function with compact support in $E_{+}^{n} \cup E^{n-1}$. Then $u$ has the following integral representation: 
1966] ASYMPTOTIC DISTRIBUTION OF EIGENVALUES AND EIGENFUNCTIONS 539

$$
u(x, t)=\int_{E_{+}^{n}} G(x, y, t)(A+t I) u(y) d y+\sum_{j=1}^{m} \int_{E^{n-1}} H_{j}(x-\hat{y}, t) B_{j} u(0, \hat{y}) d \hat{y} .
$$

$\left\{A, B_{j} ; j=1, \cdots, m\right\}$ is a uniformly regularly elliptic boundary value problem; $A$ and $B_{j}$ are defined on $E_{+}^{n}, E^{n-1}$ with infinitely differentiable coefficients. $G(x, y, t)$ is the Green's function associated with $\left\{A+t I ; B_{j} ; j=1, \cdots, m\right\}$ and is given by Theorem 4.1. The kernels $H_{j}$ are given by Lemma 4.5.

Proof. We consider the boundary value problem:

$$
\begin{aligned}
(A+t I) u(x) & =f(x) \text { on } E_{+}^{n}, \\
B_{j} u(\hat{x}) & =g_{j}(\hat{x}) \text { on } E^{n-1}, j=1, \cdots, m .
\end{aligned}
$$

Since $u$ is infinitely differentiable and has compact support in $E_{+}^{n} \cup E^{n-1}$; $f$ and $g_{i}$ also have compact supports.

We may write $u(x)$ as $u(x)=v(x)+w(x)$ where $v(x)$ is the solution of $(A+t I) v(x)=f(x)$ on $E_{+}^{n}, B_{j} v(\hat{x})=0$ on $E^{n-1}, j=1, \cdots, m$, and $w(x)$ is the solution of the boundary value problem: $(A+t I) w(x)=0$ on $E_{+}^{n}, B_{j} w(x)=g_{j}$ on $E^{n-1}, j=1, \cdots, m$. Let $G(x, y, t)$ be the Green's function associated with the elliptic boundary value problem $\left\{A+t I ; B_{j} ; j=1, \cdots, m\right\}$ given by Theorem 4.2. We get:

$$
v(x)=\int_{E_{+}^{n}} G(x, y, t) f(y) d y .
$$

Now we construct $w$. Let $H_{j}(x, y)$ be the kernels given by Lemma 4.5 ; then $w$ is given by the expression:

$$
w(x)=\sum_{j=1}^{m} \int_{E^{n-1}} h_{j}(\hat{y}) H_{j}(x-\hat{y}, t) d \hat{y} .
$$

The conclusion of the theorem follows immediately.

THEOREM 4.3. Let $\mathscr{G}(x, y, t)$ be the Green's function associated with the uniformly regularly elliptic boundary value problem $(A, \gamma)$ where $A$ is defined on $a$ bounded open subset $S$ of $E^{n}$ with infinitely differentiable coefficients; $\gamma=\left(B_{1}, \cdots, B_{m}\right)$ is a family of differential operators defined on the boundary $\Gamma$ of $S$ with infinitely differentiable coefficients. $A$ and $B_{j}$ are homogeneous differential operators.

$(A, \gamma)$ is assumed to be formally positive in the sense of Definition 2.1. Let $G(x, y, t)$ be the Green's function of Theorem 4.1 (i.e. corresponding to the case of a half space). Then:

$$
\begin{aligned}
& \mathscr{G}(x, y, t)=G(x, y, t)-u(x, y, t), \quad y \text { in } S, \\
& u(x, y, t)=\sum_{k} \tilde{u}\left(\phi^{-1}(x), \phi_{k}^{-1}(y), t\right),
\end{aligned}
$$


$\phi_{k}$ are the diffeomorphisms corresponding to the uniform regularity of $S$ and:

$$
\tilde{u}(x, y, t)=\sum_{j=1}^{m} \int_{\Gamma_{0}} H_{j}(x, \hat{z}, t) B_{j} G(y, \hat{z}, t) d \hat{z}
$$

$H_{j}(x, \hat{z}, t)$ is given by Lemma 4.5

$$
\Gamma_{0}=\left\{z: z_{1}=0 ;|z|<1\right\} .
$$

Proof. There is no loss of generality in assuming that $y=0$ is in $S$. Let $G(x, t)$ be as in Theorem 4.1. Then $G(x, t)$ is a fundamental solution of the elliptic operator $A+t I$.

If $u$ is the solution of the boundary value problem:

$$
\begin{aligned}
(A+t I) u(x) & =0 \text { on } S, \\
B_{j}(x) u & =B_{j} G(x, t) \quad \text { on } \Gamma ; j=1, \cdots, m,
\end{aligned}
$$

then $\mathscr{G}(x, t)=G(x, t)-u(x, t)$.

$S$ is a bounded domain which is uniformly regular. It may be covered by a finite number of open sets $N_{k}$ and there exists a family of infinitely differentiable unctions $\eta_{k}$ with compact supports in $N_{k}$, and such that:

$$
\sum_{k} \eta_{k}^{2}(x)=1, \quad x \text { in } S .
$$

We have:

$$
(A+t I)\left(u(x) \eta_{k}^{2}(x)\right)=\eta_{k}^{2}(x)(A+t I) u(x)+\sum_{|\alpha|+|\beta|=2 m ;|\alpha|<2 m} a_{\alpha \beta}(x) D^{\alpha} u D^{\beta} \eta_{k}^{2}(x) .
$$

Similarly for $B_{j}\left(u \eta_{k}^{2}\right)$.

We consider the boundary value problem:

$$
\begin{aligned}
(A+t I) u \eta_{k}^{2} & =f_{k} \text { on } N_{k} \cap S, \\
B_{j}\left(u \eta_{k}^{2}\right) & =g_{j k}+h_{j k} \text { on } N_{k} \cap \Gamma ; j=1, \cdots, m,
\end{aligned}
$$

where:

$$
\begin{aligned}
& f_{k}(x)=\sum_{|\alpha|<2 m ;|\alpha|+|\beta|=2 m} a_{\alpha \beta}(x) D^{\alpha} u D^{\beta} \eta_{j}^{2}(x), \\
& h_{j k}(x)=\sum_{|\alpha|<r j ;|\alpha|+|\beta|=r_{j}} b_{j \beta k}(x) D^{\alpha} u D^{\beta} \eta_{k}^{2}(x) .
\end{aligned}
$$

Using the diffeomorphisms $\phi_{k}(x)$ we map $N_{k}$ into the positive half ball. Set: $\tilde{u}_{k}(x)=\left(\eta_{k}^{2}(u)\right)\left(\phi_{k}(x)\right) ; \tilde{f}_{k}(x) ; \tilde{g}_{j k}(x) ; \tilde{h}_{j k}$ are similarly defined.

Using the same notations for the transplanted operators, we get:

$$
\begin{aligned}
(A+t I) \tilde{u}_{k}(x) & =\tilde{f}_{k}(x) \quad \text { on } B^{+}=\left\{x: x_{1}>0 ;|x|<1\right\}, \\
B_{j} \tilde{u}(0, \hat{x}) & =\tilde{g}_{j k}(0, \hat{x})+\tilde{h}_{j k}(0, \hat{x}), \quad \text { on } \Gamma ; j=1, \cdots, m .
\end{aligned}
$$


$\tilde{f}_{k}$ is an infinitely differentiable function with compact support in $B^{+} \cup \Gamma_{0}$. Applying Theorem 4.2; we obtain:

$$
\tilde{u}_{k}(x)=\int_{B^{+}} \tilde{f}_{k}(y) G(x, y, t) d y+\sum_{j=1}^{m} \int_{\Gamma_{0}} H_{j}(x-\hat{y}, t)\left\{\tilde{g}_{j k}(\hat{y})+\tilde{h}_{j k}(\hat{y})\right\} d \hat{y} .
$$

Since $\Sigma_{k} \eta_{k}^{2}(x)=1$, we have:

$$
\tilde{u}(x)=\sum_{k} \tilde{u}_{k}(x)=\sum_{j=1}^{m} \int_{\Gamma_{0}} H_{j}(x-\hat{y}, t) B_{j} G(0, \hat{y}, t) d \hat{y} .
$$

The theorem is proved.

5. THEOREM 5.1. Let $\mathscr{G}_{2 p}$ be the 2 pth iterate of the Green's function $\mathscr{G}$ defined in Theorem 4.3. Let $A_{\gamma}$ be the realization of $A$ under null boundary conditions $\gamma$ as an operator on $L^{2}(S)$. If $(A, \gamma)$ is formally self-adjoint and $\lambda_{j}, \phi_{j}$ are respectively the eigenvalues and eigenfunctions of $A_{\gamma}$; then: for $2 m p>n$ :

$$
D_{x}^{\alpha} D_{y}^{\beta} \mathscr{G}_{2 p}(x, y, t)=\sum_{j} D^{\alpha} \phi_{j}(x) D^{\beta} \phi_{j}(y)\left(\lambda_{j}+t\right)^{-2 p} ; \quad|\alpha|,|\beta| \leqq 2 m .
$$

Proof. Let $2 m p>n$, then $\left(A_{\gamma}+t I\right)^{-p}$ is of Hilbert-Schmidt type. Since $(A, \gamma)$ is formally self-adjoint, it follows from Theorem 2.1 that $A_{\gamma}$ is self-adjoint; $\lambda_{j}+t>0$, we have a complete orthonormal system of eigenfunctions $\phi_{j}$.

Consider:

$$
\left(\left(A_{\gamma}+t I\right)^{-p} f, \phi_{j}\right)=\int_{S} \int_{S} \mathscr{G}_{(p)}(x, y, t) f(y) \phi_{j}(z) d y d z, f \text { in } L^{2}(S) .
$$

We get:

$$
\left(\lambda_{j}+t\right)^{-p} \phi_{j}(y)=\int_{S} \mathscr{G}_{(p)}(z, y, t) \phi_{j}(z) d z .
$$

Using Parseval's formula, we obtain:

$$
\begin{aligned}
\sum_{j}\left(\lambda_{j}+t\right)^{-2 p} \phi_{j}(x) \phi_{j}(y) i & =\int_{S} \mathscr{G}_{(p)}(z, x, t) \mathscr{G}_{(p)}(z, y, t) d z \\
& =\mathscr{G}_{(2 p)}(x, y, t) .
\end{aligned}
$$

Let $\mathscr{G}_{2 p, k}(x, y, t)=\sum_{j=1}^{k}\left(\lambda_{j}+t\right)^{-2 p} \phi_{j}(x) \phi_{j}(y)$ then:

$$
\begin{aligned}
&\left\|\mathscr{G}_{2 p, k}-\mathscr{G}_{2 p}\right\|_{W^{2 m, 2} \times W^{2 m, 2}} \leqq \sum_{k}^{l}\left(\lambda_{j}+t\right)^{-2 p}\left\|\phi_{j}\right\|_{W^{2 m, 2}}, \\
& \leqq \sum_{k}^{l}\left(\lambda_{j}+t\right)^{-2 p+2} \rightarrow 0 \text { as } l, k \rightarrow \infty, \\
& \mathscr{G}_{2 p, k}(x, y, t) \rightarrow \mathscr{G}_{(2 p)}(x, y, t) \text { in } W^{2 m, 2} \times W^{2 m, 2} .
\end{aligned}
$$


In particular; $D_{x}^{\alpha} D_{y}^{\beta} \mathscr{G}_{2 p, k}(x, y, t) \rightarrow D_{x}^{\alpha} D_{y}^{\beta} \mathscr{G}_{2 p}(x, y, t)$ in $L^{2} \times L^{2}$ and we get:

$$
D_{x}^{\alpha} D_{y}^{\beta} \mathscr{G}_{2 p}(x, y, t)=\sum_{j}\left(\lambda_{j}+t\right)^{-2 p} D^{\alpha} \phi_{j}(x) D^{\beta} \phi_{j}(y) .
$$

LeMma 5.1. Let $\mathscr{G}_{p}(x, y, t)$ be the pth iterate of the Green's function defined in Theorem 4.3. Then if $2 m p>n+|\alpha|+|\beta|$,

$$
\begin{aligned}
& x \neq y, t^{p-(n+|\alpha|+|\beta|) / 2 m} D_{x}^{\alpha} D_{y}^{\beta} \mathscr{G}_{p}(x, y, t) \rightarrow 0 \text { as } t \rightarrow+\infty, \\
& t^{p-(n+|\alpha|+|\beta|) / 2 m} D^{\alpha+\beta} \mathscr{G}_{p}(x, x, t)=(2 \pi)^{-n} \int_{E^{n}} \xi^{\alpha+\beta}[a(x, \xi)+1]^{-p} d \xi
\end{aligned}
$$

as $t \rightarrow+\infty$; for $x, y$ in $S$.

Proof. We prove the lemma for $|\alpha|=|\beta|=0$; the general case may be treated in the same fashion. Let $G$ be the Green's function associated with the elliptic boundary value problem $\{A+t I ; \gamma\}$ on a half space with infinitely differentiable coefficients. From Theorem 4.2, we have

$G(x, y, t)=G_{y}(x, y, t)+\int_{B^{+}} G_{z}(x, z, t) v(z, y, t) d z+\sum_{j=1}^{m} \int_{\Gamma_{0}} H_{j z}(x-\hat{z}, t) h_{j}(\hat{z}, \hat{y}) d \hat{z}$ $x, y$ in $B^{+}$.

$G_{y}$ is the Green's function associated with the constant coefficients problem $\left\{A_{y}+t I ; B_{j y} ; j=1, \cdots, m\right\}$ on a half space.

We show that:

$$
\lim _{t \rightarrow+\infty} t^{p-n / 2 m} G_{p}(x, y, t)=\lim _{t \rightarrow+\infty} t^{p-n / 2 m} G_{p, y}(x, y, t) .
$$

First consider the case $2 m>n$. With $t=\tau^{2 m}$, we have:

$$
\begin{gathered}
\tau^{2 m-n} \int_{B^{+}} G_{z}(x, z, t) v(y, z, t) d z=O(1) \tau^{-\varepsilon} \int_{B^{+}}|y-z|^{1-n-\varepsilon}\left(1+|\tau(x-y)|^{N}\right)^{-1} d z, \\
\tau^{2 m-n} \int_{\Gamma_{0}} H_{j \hat{z}}(x-\hat{z}, t) h_{j}(\hat{z}, y, t) d \hat{z}=O(1) \tau^{-\varepsilon}\left|x_{1}\right|^{-n} \int_{\Gamma_{0}}|\hat{z}-\hat{y}|^{-n+2-\varepsilon}\left(1+|\tau(\hat{z}-\hat{y})|^{N}\right)^{-1} d \hat{z} .
\end{gathered}
$$

So:

$$
\lim _{t \rightarrow+\infty} t^{1-n / 2 m} G(x, y, t)=\lim _{t \rightarrow+\infty} t^{1-n / 2 m} G_{(y)}(x, y, t) .
$$

Now if $2 m<n$, from Theorem 4.1; we have:

so

$$
G(x, y, t)=G_{(y)}(x, y, t) \quad\left(1+O(1) \tau^{-\varepsilon}\right)
$$

$$
\lim _{t \rightarrow+\infty} t^{p-n / 2 m} G_{(p)}(x, y, t)=\lim _{t \rightarrow+\infty} t^{p-n / 2 m} G_{(p), y}(x, y, t) .
$$

On the other hand: 
with

$$
\mathscr{G}(x, y, t)=G(x, y, t)+\sum_{k} \tilde{v}\left(\phi_{k}^{-1}(x), \phi_{k}^{-1}(y), t\right)
$$

$$
\tilde{v}(x, y, t)=\sum_{j=1}^{m} \int_{\Gamma_{0}} H_{j}(x-\hat{z}, t) B_{j} G(\hat{z}, y, t) d \hat{z} .
$$

An argument as above gives:

$$
\lim _{t \rightarrow+\infty} t^{p-n / 2 m} \mathscr{G}_{(p)}(x, y, t)=\lim _{t \rightarrow+\infty} t^{p-n / 2 m} G(x, y, t) .
$$

The conclusion of the lemma follows from Theorem 3.4 .

THEOREM 5.2. Let $A_{\gamma}$ be the realization of the positively strongly elliptic operator $A$ under null boundary conditions $\gamma=\left(B_{1}, \cdots, B_{m}\right)$ as an operator on $L^{2}(S)$. The operators $A$ and $B_{j}$ are defined on a bounded open set $S$ and on the boundary $\Gamma$ respectively and have infinitely differentiable coefficients.

$(A, \gamma)$ is assumed to be uniformly regularly elliptic, formally self-adjoint and formally positive in the sense of Definition 2.1. Let $\lambda_{j}, \phi_{j}$ be the eigenvalues and eigenfunctions of $A_{\gamma}$. Then:

$$
N(t)=\sum_{\lambda_{j} \leqq t} 1 \sim(2 \pi)^{-n} t^{n / 2 m} \int_{S} \int_{a(x, \xi)<1} d \xi d x \text { as } t \rightarrow+\infty .
$$

$$
t^{-(n+|\alpha|+|\beta|) \mid 2 m} D_{x}^{\alpha} D_{y}^{\beta} e(x, y, t)=t^{-(n+|\alpha|+|\beta|)} \sum_{\lambda_{j} \leqq t} D^{\alpha} \phi_{j}(x) D^{\beta} \phi_{j}(y) \rightarrow 0
$$

as $t \rightarrow \infty$ for $x, y$ in $S$ and $x \neq y$.

(3) $D^{\alpha+\beta} e(x, x, t) \sim(2 \pi)^{-n} t^{(n+|\alpha|+|\beta|) / 2 m} K(n, m, p, \alpha, \beta) \int_{E^{n}} \xi^{\alpha+\beta}[a(x, \xi)+1]^{-2 p} d \xi$

as $t \rightarrow \infty$ for $x$ in $S$ and $4 m p>n+|\alpha|+|\beta|$.

$$
K(n, m, p, \alpha, \beta)=\frac{\Gamma(2 p)}{\Gamma\left(1+\frac{n}{2 m}\right) \Gamma\left(2 p-\frac{n+|\alpha|+|\beta|}{2 m}\right)} .
$$

Proof. One can show easily that:

$$
\sum_{j}\left(\lambda_{j}+t\right)^{-2 p}=\int_{S} \mathscr{G}_{(2 p)}(x, x, t) d x .
$$

Consider the sequence of integrable functions $t^{2 p-n / 2 m} \mathscr{G}_{(2 p)}(x, x, t)$. For large $t$, we have from the previous lemmas: $t^{2 p-n / 2 m}\left|\mathscr{G}_{(2 p)}(x, x, t)\right| \leqq M$ for all $x$ in $S$ and $M$ is a constant independent of $x$ and $t$. We apply the Lebesgue dominated convergence theorem and we get: 


$$
t^{2 p-n / 2 m} \int_{S} \mathscr{G}_{(2 p)}(x, x, t) d x \sim(2 \pi)^{-n} \int_{S} \int_{E^{n}}[a(x, \xi)+1]^{-2 p} d \xi d x
$$

as $t \rightarrow+\infty$.

Applying the Tauberian theorem of Hardy-Littlewood [10], we get the results for $N(t)$.

We may write:

$$
\mathscr{G}_{(2 p)}(x, y, t)=\sum_{j}\left(\lambda_{j}+t\right)^{-2 p} \phi_{j}(x) \phi_{j}(y)=\int_{0}^{\infty}(\lambda+t)^{-2 p} d e(x, y, \lambda)
$$

where $e(x, y, t)$ is the spectral function. Taking into account the results of Lemma 5.1 and applying the Tauberian theorem of Hardy-Littlewood again, we get the results stated in the theorem.

6. The case of a nonself-adjoint regular elliptic boundary value problem is considered. The study of the asymptotic distribution of eigenvalues for the nonself-adjoint case has been carried out by Carleman [8] and Keldych [11] for second order elliptic equations.

THEOREM 6.1. Let $\left\{A ; B_{j} ; j=1, \cdots, m\right\}$ be a uniformly regularly elliptic boundary value problem where $A$ and $B_{j}$ are defined on a bounded open subset $S$ of $E^{n}$ and on the boundary $\Gamma$ with infinitely differentiable coefficients. $(A, \gamma)$ with $\gamma=\left(B_{1}, \cdots, B_{m}\right)$ is assumed to be formally positive in the sense of Definition 2.1. Let $A_{\gamma}$ be the realization of $A$ under null boundary conditions $\gamma$ as an operator $L^{2}(S)$. If $2 m p>n$ where $2 m$ is the order of $A$, the operator $\left(A_{\gamma}+t I\right)^{-2 p}$ is of trace class. Let $\lambda_{j}$ be the eigenvalues of $A$, then:

$$
\operatorname{tr}(A+t I)^{-2 p}=\sum_{j}\left(\lambda_{j}+t\right)^{-2 p}=\int_{S} \mathscr{G}_{(2 p)}(x, x, t) d x .
$$

$\mathscr{G}_{(2 p)}(x, y, t)$ is the 2 pth iterate of the Green's function associated with $A_{\gamma}+t I$ on $S$.

Proof. With the above hypothesis, it has been proved in $\$ 2$ that $\left(A_{\gamma}+t I\right)^{-p}$ is of Hilbert-Schmidt type, so $\left(A_{\gamma}+t I\right)^{-2 p}$ is of trace class. Let $\phi_{j}$ be the generalized eigenfunctions of $A_{\gamma}$. They form an orthonormal basis in $L^{2}(S)$. Denote by $P_{j}$ the orthogonal projection of $L^{2}(S)$ onto the subspace of $L^{2}(S)$ spanned by $\left\{\phi_{1}, \cdots, \phi_{j}\right\}$; consider the operator: $T_{j}=P_{j}(A+t I)^{-2 p} P_{j}$.

It takes the subspace spanned by $\left\{\phi_{1}, \cdots, \phi_{j}\right\}$ into itself. The subspace is of finite dimension and we have:

$$
\begin{aligned}
\operatorname{tr}\left(T_{j}\right) & =\sum_{k=1}^{j}\left(\lambda_{k}+t\right)^{-2 p}, \\
\operatorname{tr}\left(T_{n}-T_{m}\right) & =\operatorname{tr}\left(T_{n}\right)-\operatorname{tr}\left(T_{m}\right)=\sum_{j=m}^{n}\left(\lambda_{j}+t\right)^{-2 p} .
\end{aligned}
$$


1966] ASYMPTOTIC DISTRIBUTION OF EIGENVALUES AND EIGENFUNCTIONS 545

Denote by $\|T \mid\|$ the trace norm of an operator of finite rank. (Ruston [13].) Then:

$$
\left\|\left|T_{n}-T_{m}\right|\right\| \leqq \sum_{m}^{n}\left|\lambda_{j}+t\right|^{-2 p} .
$$

Since $\left(A_{\gamma}+t I\right)^{-p}$ is of Hilbert-Schmidt type: $\Sigma_{j}\left|\lambda_{j}+t\right|^{-2 p}<\infty$. It follows that $T \rightarrow \mathscr{C}$ in the trace norm topology and:

$$
\operatorname{tr}(\mathscr{C}) \sum_{j}\left(\lambda_{j}+t\right)^{-2 p}
$$

We now show that $\mathscr{C}=\left(A_{\gamma}+t I\right)^{-2 p}$. We know that $\left(A_{\gamma}+t I\right)^{-2 p}$ is a compact operator and $T_{j} \rightarrow\left(A_{\gamma}+t I\right)^{-2 p}$ in the operator norm topology. Since $T_{j} \rightarrow \mathscr{I}$ in the trace norm, it converges to $\tau$ in the operator norm; hence $\mathscr{C}=\left(A_{\gamma}+t I\right)^{-2 p}$ and:

$$
\operatorname{tr}\left(A_{\gamma}+t I\right)^{-2 p}=\sum_{j}\left(\lambda_{j}+t\right)^{-2 p}
$$

We may write $\mathscr{C}=\tau_{R}+i \mathscr{C}_{1}$ where $\mathscr{C}_{R}=\left(\mathscr{C}^{*}-\mathscr{C}\right) / 2, \mathscr{C}_{1}=\left(\mathscr{C}-\mathscr{C}^{*}\right) / 2 i$, Since $\mathscr{C}_{R}, \mathscr{C}_{I}$ are self-adjoint, we may apply results of $\S 5$ to get

$$
\operatorname{tr}\left(A_{\gamma}+t I\right)^{-2 p}=\sum_{j}\left(\lambda_{j}+t\right)^{-2 p}=\int_{S} \mathscr{G}_{(2 p)}(x, x, t) d x .
$$

THEOREM 6.2. Let $(A, \gamma)$ be a uniformly regularly elliptic boundary value problem with infinitely differentiable coefficients and formally positive in the sense of Definition 2.1. If $A_{\gamma}$ is the realization of the positively strongly elliptic operator $A$ as an operator on $L^{2}(S)$ under null boundary conditions $\gamma$ and $\lambda_{j}$ are the eigenvalues of $A_{\gamma}$ then:

$$
N(t)=\sum_{\operatorname{Re} \lambda_{j} \leqq t} 1 \sim(2 \pi)^{-n} t^{n / 2 m} w_{a}(S) \text { as } t \rightarrow+\infty
$$

where $w_{a}(S)=\int_{S} w_{a}(x) d x$ and $w_{a}(x)=\int_{a(x, \xi)<1} d \xi$.

Proof. Set $\lambda_{j}=\alpha_{j}+i \beta_{j} ; f(t)=\Sigma_{j}\left(\alpha_{j}+t\right)^{-2 p}$ and $g(t)=\Sigma_{j}\left(\lambda_{j}+t\right)^{-2 p}$.

Let $h(t)=f(t)-g(t)=\Sigma_{j}\left\{\left(\alpha_{j}+t\right)^{-2 p}-\left(\lambda_{j}+t\right)^{-2 p}\right\}$.

It has been proved by Browder [4] that the spectrum of $A_{\gamma}$ is contained inside an algebraic curve $|\operatorname{Im} \zeta| \leqq c(\operatorname{Re} \zeta)^{\mu}$ with $\mu=(2 m-1) / 2 m$, we get:

$$
|h(t)| \leqq \sum_{j}\left(\alpha_{j}+t\right)^{-2 p-1}\left|\alpha_{j}\right|^{\mu}
$$

The eigenvalues have an accumulation point at infinity, hence there exists a number $N$ such that:

We have:

$$
\left|\alpha_{N}\right|<t^{\delta} \leqq \alpha_{N+1}, \quad 0<\delta<1
$$

$$
\sum_{N+1}\left|\alpha_{j}\right|^{\mu}\left(\alpha_{j}+t\right)^{-2 p-1} \leqq \sum_{N+1} t^{(\mu-1) \delta}\left(\alpha_{j}+t\right)^{-2 p}, \quad|h(t)| \leqq c t^{(\mu-1) \delta} f(t) .
$$


It follows that: $\lim t^{2 p-n / 2 m} \sum_{j}\left(\alpha_{j}+t\right)^{-2 p}=\lim t^{2 p-n / 2 m} \sum_{j}\left(\lambda_{j}+t\right)^{-2 p}$.

By an argument as in Theorem 5.2 and applying the Tauberian theorem of Hardy-Littlewood we get:

$$
N(t)=\sum_{\operatorname{Re} \lambda \leqq t} 1 \sim t^{n / 2 m} w_{a}(S) \cdot(2 \pi)^{-n} \text { as } t \rightarrow+\infty
$$

\section{BIBLIOGRAPHY}

1. S. Agmon, On the eigenfunctions and on the eigenvalues of general elliptic boundary value problem, Comm. Pure Appl. Math. 15 (1962), 119-147.

2. S. Agmon, A. Douglis and L. Nirenberg, Estimates near the boundary for solutions of elliptic partial differential equations satisfying general boundary conditions. I, Comm. Pure Appl. Math. 12 (1959), 623-727.

3. F. Browder, Estimates and existence theorems for elliptic boundary value problems, Proc. Nat. Acad. Sci. U.S.A. 45 (1959), 365-372.

4. - The spectral theory of strongly elliptic differential operators, Proc. Nat. Acad. Sci. U.S.A. 45 (1959), 1413-1422.

5. - On the spectral theory of elliptic differential operators. I, Math. Ann. 142 (1961), 22-127.

6. - A priori estimates for solutions of elliptic boundary value problems. I, II, Indag. Math. 22 (1960), 145-169.

7. T. Carleman, Propriétés asymptotiques des fonctions fondamentales des membranes vibrantes, Attonde Skand. Matematikerekongrussen, Stockholm, 1934, pp. 34-44.

8. —_ Uber die symptotische verteilung der Eigenwerte partieller Differentialgleichungen, Ber. Verh. Sächs. Akad. Wiss. Leipzig Math.-Nat. Kl. 88 (1936), 119-132.

9. L. Gårding, On the asymptotic distribution of the eigenvalues and eigenfunctions of elliptic differential operators, Math. Scand. 1 (1953), 237-255.

10. G. H. Hardy and T. E. Littlewood, Notes on the theory of series (XI): On Tauberian theorems, Proc. London Math. Soc. (2) 30 (1930), 23-27.

11. M. V. Keldych, On the eigenvalues and eigenfunctions of some classes of non-self-adjoint equations, Dokl. Akad. Nauk SSSR 77 (1951), 11-14.

12. E. E. Levi, Sulle equationi lineari totalmette ellittiche alle derivate parziali, Rend. Circ Mat. Palermo. 24 (1907), 275-317.

13. A. F. Ruston, The Fredholm theory of integral equations for equations belonging to the trace class of a general Banach space, Proc. London Math. Soc. 53 (1951), 109-124.

14. M. Schechter, General boundary value problems for elliptic partial differential equations, Comm. Pure Appl. Math. 12 (1959), 457-486.

15. S. Agmon, On kernels and eigenfunctions of operators related to elliptic problems, Comm. Pure Appl. Math. 18 (1965), 559-579(2).

16. F. Browder, Asymptotic distribution of eigenvalues and eigenfunctions for non-local elliptic boundary value problems. I, Amer. J. Math. 57 (1965), 175-195(2).

\section{UNIVERSITY OF MONTREAL, MONTREal, CANADA}

(2) Added in proof. 